ANNALES

POLONICI MATHEMATICI

$85.2(2005)$

\title{
Strongly starlike and spirallike functions
}

\author{
by ADAm Lecko (Rzeszów)
}

\begin{abstract}
The aim of this paper is to present a new method of proof of an analytic characterization of strongly starlike functions of order $(\alpha, \beta)$. The relation between strong starlikeness and spirallikeness of the same order is discussed in detail. Some well known results are reproved.
\end{abstract}

\section{Introduction}

0.1. In this paper we study the concepts of strong starlikeness and spirallikeness with respect to a finite point as well as with respect to infinity. We examine the classes $\mathcal{S}^{*}(\alpha, \beta ; \xi)$ and $\mathcal{S}^{\supset}(\alpha, \beta ; \xi)$, where $\alpha, \beta \in[0,1], \xi \in \mathbb{D}$, of strongly starlike and spirallike functions of order $(\alpha, \beta)$, respectively. Moreover we study the classes $\Sigma^{*}(\alpha, \beta)$ and $\Sigma^{\supset}(\alpha, \beta)$, where $\alpha, \beta \in[0,1]$, of meromorphic strongly starlike and meromorphic spirallike functions of or$\operatorname{der}(\alpha, \beta)$, respectively.

The class $\mathcal{S}^{*}(\alpha, \beta ; \xi)$ was studied in [L], [LL] and [LS]; here we present new methods of proofs and new results. Some well known results are reproved in a new elementary way. We discuss the relation between strong starlikeness and spiralikeness of the same order in detail.

Let us emphasize that we are interested in turning geometrical ideas into analytical formulas. We define strong starlikeness and spirallikeness as geometrical properties of plane domains. Next we find analytic formulas for the corresponding classes of univalent functions. As we remark below, strong starlikeness was usually defined by various authors as a property of analytic functions given by analytic formulas like $(0.1)$ or $(0.2)$ below. Next the authors searched for geometrical characterizations of the functions thus defined.

0.2. Our starting point is the notion of strong starlikeness of order $\alpha$. The class $\mathcal{S}^{*}(\alpha ; 0), \alpha \in(0,1]$, of analytic functions $f$ with $f(0)=0$ and

2000 Mathematics Subject Classification: Primary 30C45.

Key words and phrases: strongly starlike functions, spirallike functions, meromorphic strongly starlike functions, meromorphic spirallike functions. 
$f^{\prime}(0) \neq 0$ satisfying the condition

$$
\left|\arg \left\{\frac{z f^{\prime}(z)}{f(z)}\right\}\right|<\alpha \frac{\pi}{2}, \quad z \in \mathbb{D},
$$

and usually called strongly starlike of order $\alpha$, was introduced independently by Brannan and Kirwan [BK] and Stankiewicz [S1-2] (see also [G, Vol. I, pp. 138-139]). Brannan and Kirwan found a geometric condition called $\delta$ visibility which is sufficient for a function to be in $\mathcal{S}^{*}(\alpha ; 0)$. Stankiewicz [S2] obtained an external geometric characterization of strongly starlike functions. Ma and Minda $[\mathrm{MM}]$ presented an internal geometric characterization of functions in $\mathcal{S}^{*}(\alpha ; 0)$ using the concept of $k$-starlike domains.

The second source of our interest is a generalization of strong starlikeness given by Bucka and Ciozda [BC] (see also [G, Vol. I, p. 141]). They defined, for $\alpha, \beta \in(0,2]$, the class of analytic functions $f$ with $f(0)=0$ and $f^{\prime}(0) \neq 0$ by the condition

$$
-\beta \frac{\pi}{2}<\arg \left\{\frac{z f^{\prime}(z)}{f(z)}\right\}<\alpha \frac{\pi}{2}, \quad z \in \mathbb{D} .
$$

This class coincides with $\mathcal{S}^{*}(\alpha, \beta ; 0)$ (defined in 3.1 ) when $\alpha$ and $\beta$ are restricted to the interval $(0,1]$.

0.3. Some results presented here were published in [L], [LL] and [LS] but the proofs given here are new. Theorem 3.2 was published in [LL] but now we reprove it afresh. The case $\beta=\alpha$, i.e. the class $\mathcal{S}^{*}(\alpha, \alpha ; \xi)$ was studied in $[\mathrm{L}]$. Other results presented here have not been published before.

\section{Preliminaries}

1.1. For $z_{0} \in \mathbb{C}$ and $r>0$ let $\mathbb{D}\left(z_{0}, r\right)=\left\{z \in \mathbb{C}:\left|z-z_{0}\right|<r\right\}$. For $r>0$ let $\mathbb{D}_{r}=\mathbb{D}(0, r)$ and $\mathbb{D}_{r}^{*}=\{z \in \mathbb{C}:|z|>r\} \cup\{\infty\}$. Let $\mathbb{D}=\mathbb{D}_{1}$, $\mathbb{D}^{*}=\mathbb{D}_{1}^{*}$ and $\mathbb{T}=\partial \mathbb{D}$.

1.2. Define

$$
\hbar(z)= \begin{cases}1 / z, & z \in \overline{\mathbb{C}} \backslash\{0, \infty\} \\ 0, & z=\infty \\ \infty, & z=0\end{cases}
$$

1.3. For $A, B \subset \mathbb{C}$ and $w \in \mathbb{C}$ we define

$$
A B=\{u v \in \mathbb{C}: u \in A \wedge v \in B\}, \quad w A=\{w\} A .
$$

For $A \subset \overline{\mathbb{C}}$, let

$$
A^{-1}=\{\hbar(u): u \in A\} .
$$

Let $\bar{A}$, Int $A$ and Ext $A$ denote the closure, interior and exterior of the set $A \subset \overline{\mathbb{C}}$, respectively. 
1.4. For any $\xi \in \mathbb{D}$ define the Möbius transformation

$$
\varphi_{\xi}(z)=\frac{z-\xi}{1-\bar{\xi} z}, \quad z \in \mathbb{C} \backslash\{1 / \bar{\xi}\} .
$$

For every $\xi \in \mathbb{D}, \varphi_{\xi}$ is an analytic automorphism of $\mathbb{D}$.

1.5. Let $\mathbb{B}(\xi, \rho), \xi \in \mathbb{D}, \rho>0$, denote the hyperbolic open disk with hyperbolic center at $\xi$ and hyperbolic radius $\rho$. We recall that

$$
\mathbb{B}(\xi, \rho)=\left\{z \in \mathbb{D}:\left|\varphi_{\xi}(z)\right|<\tanh \rho\right\} .
$$

For fixed $\xi \in \mathbb{D}$ let $C_{\rho}=\partial \mathbb{B}(\xi, \rho)$. We will use the following parametrization of $C_{\rho}$ :

$$
C_{\rho}: \quad z=c_{\rho}(\theta)=\frac{R e^{i \theta}+\xi}{1+\bar{\xi} R e^{i \theta}}, \quad \theta \in[0,2 \pi),
$$

where $R=\tanh \rho \in(0,1)$. Clearly, $C_{\rho}$ is positively oriented.

1.6. The set of all analytic functions in $\mathbb{D}$ is denoted by $\mathcal{A}$. Its subset of univalent functions in $\mathbb{D}$ is denoted by $\mathcal{S}$. For fixed $\xi \in \mathbb{D}$ the set of all functions $f \in \mathcal{A}$ such that $f(\xi)=0$ will be denoted by $\mathcal{A}(\xi)$. Set $\mathcal{S}(\xi)=$ $\mathcal{A}(\xi) \cap \mathcal{S}$.

1.7. Let $\Sigma$ consist of all functions $g$ of the form

$$
g(\zeta)=b \zeta+\sum_{n=0}^{\infty} \frac{b_{n}}{\zeta^{n}}, \quad \zeta \in \mathbb{D}^{*}, b \in \mathbb{C} \backslash\{0\},
$$

univalent in $\mathbb{D}^{*}$. Let $\Sigma^{\prime}$ be the class of $g \in \Sigma$ such that $g(\zeta) \neq 0$ for $\zeta \in \mathbb{D}^{*}$. Recall that there exists a one-to-one correspondence between $\mathcal{S}(0)$ and $\Sigma^{\prime}$. If $f \in \mathcal{S}(0)$, then $g(\zeta)=\hbar \circ f \circ \hbar(\zeta), \zeta \in \mathbb{D}^{*}$, belongs to $\Sigma^{\prime}$. Conversely, if $g \in \Sigma^{\prime}$, then $f(z)=\hbar \circ g \circ \hbar(z), z \in \mathbb{D}$, belongs to $\mathcal{S}(0)$ (see e.g. [P2, p. 8]).

\section{Strongly starlike domains of order $(\alpha, \beta)$}

2.1. For $k, l \in(0,2]$ let $K_{1}(k)$ and $K_{2}(l)$ be the closed disks of radius $1 / k$ and $1 / l$ centered at $1 / 2-i \sqrt{1 / k^{2}-1 / 4}$ and $1 / 2+i \sqrt{1 / l^{2}-1 / 4}$, respectively. Moreover let, $K_{1}(0)=\{v \in \mathbb{C}: \operatorname{Re} v<0\} \cup[0,1], K_{2}(0)=\{v \in \mathbb{C}: \operatorname{Re} v>0\}$ $\cup[0,1]$. For $k, l \in[0,2]$ let $E_{k, l}=K_{1}(k) \cap K_{2}(l)$. For $k, l \in[0,2]$ define

$$
\Gamma_{k}^{+}=\partial E_{k, l} \cap \partial K_{1}(k), \quad \Gamma_{l}^{-}=\partial E_{k, l} \cap \partial K_{2}(l) .
$$

Thus $\Gamma_{k}^{+}$for $k \in(0,2]$ and $\Gamma_{l}^{-}$for $l \in(0,2]$ are closed circular arcs with end points 0 and 1 lying in the closed upper and lower half-plane, respectively. Moreover, $\Gamma_{0}^{+}=\Gamma_{0}^{-}=[0,1]$. An easy calculation shows that $\Gamma_{k}^{+}$and $\Gamma_{l}^{-}$ for $k, l \in[0,2]$ make angles of $\pi / 2-\arccos (k / 2)$ and $\pi / 2-\arccos (l / 2)$ to the real axis at 1 and 0 , respectively. Thus $E_{k, l}$ is a set bounded by $\Gamma_{k}^{+}$and $\Gamma_{l}^{-}$which make an angle of $\pi-(\arccos (k / 2)+\arccos (l / 2))$ to each other. 
Clearly, $E_{0,0}=[0,1], E_{2,2}=\overline{\mathbb{D}(1 / 2,1 / 2)}, E_{0,2}$ and $E_{2,0}$ are half-disks. For $k \in(0,2)$ or $l \in(0,2), E_{k, l}$ is a lens-shape set.

2.2. It is convenient to parametrize $\Gamma_{k}^{+} \backslash\{0\}, k \in(0,2]$, and $\Gamma_{l}^{-} \backslash\{0\}$, $l \in(0,2]$, in polar coordinates:

$$
\begin{array}{lll}
\Gamma_{k}^{+} \backslash\{0\}: & z=\varrho_{k}^{+}(\theta) e^{i \theta}, & \theta \in[0, \pi / 2-\arccos (k / 2)), \\
\Gamma_{l}^{-} \backslash\{0\}: & z=\varrho_{l}^{-}(\theta) e^{i \theta}, & \theta \in(-\pi / 2+\arccos (l / 2), 0],
\end{array}
$$

where

$$
\varrho_{k}^{+}(\theta)=\cos \theta-\left(\sqrt{\frac{4}{k^{2}}-1}\right) \sin \theta, \quad \varrho_{l}^{-}(\theta)=\cos \theta+\left(\sqrt{\frac{4}{l^{2}}-1}\right) \sin \theta .
$$

Thus $\Gamma_{k}^{+}$and $\Gamma_{l}^{-}$are oriented from 1 to 0 and from 0 to 1 , respectively. This means that the boundary of $E_{k, l}$, for $k \in(0,2]$ or $l \in(0,2]$, is positively oriented. The line segment $\Gamma_{0}^{+}=[0,1]$ will be oriented from 1 to 0 , and $\Gamma_{0}^{-}=[0,1]$ from 0 to 1 .

For $w \in \mathbb{C} \backslash\{0\}, w \Gamma_{k}^{+}$is oriented from $w$ to 0 , and $w \Gamma_{l}^{-}$from 0 to $w$. This means that the boundary of $w E_{k, l}$ for $k \in(0,2]$ or $l \in(0,2]$ is positively oriented.

For every $z \in \Gamma_{k}^{+} \backslash\{0,1\}, k \in[0,2]$, we denote by $\varkappa(z) \in[0, \pi / 2]$ the directed angle from $i z$ to the tangent vector to $\Gamma_{k}^{+}$at $z$. We also set $\varkappa(1)=$ $\lim _{\Gamma_{k}^{+} \ni z \rightarrow 1} \varkappa(z)$ and $\varkappa(0)=\lim _{\Gamma_{k}^{+} \ni z \rightarrow 0} \varkappa(z)$. For $k \in(0,2]$ from (2.1) we have

$$
\begin{aligned}
& \varkappa(z)=\varkappa\left(\varrho_{k}^{+}(\theta) e^{i \theta}\right)=\arg \frac{\frac{d}{d \theta}\left(\varrho_{k}^{+}(\theta) e^{i \theta}\right)}{i \varrho_{k}^{+}(\theta) e^{i \theta}} \\
& =\arg \left\{-i \frac{\frac{k}{2} \sin \theta+\sqrt{1-\frac{k^{2}}{4}} \cos \theta}{-\frac{k}{2} \cos \theta+\sqrt{1-\frac{k^{2}}{4}} \sin \theta}+1\right\} \\
& =\arg \{i \tan (\arccos (k / 2)+\theta)+1\}=\arccos (k / 2)+\theta
\end{aligned}
$$

for $\theta \in(0, \pi / 2-\arccos (k / 2))$. Moreover, $\varkappa(1)=\arccos (k / 2)$ and $\varkappa(0)=\pi / 2$.

Similarly, for every $z \in \Gamma_{l}^{-} \backslash\{0,1\}, l \in[0,2]$, we denote by $\vartheta(z) \in$ $[-\pi / 2,0]$ the directed angle from $i z$ to the tangent vector to $\Gamma_{l}^{-}$at $z$ and we set $\vartheta(1)=\lim _{\Gamma_{l}^{-} \ni z \rightarrow 1} \vartheta(z)$. We also set $\vartheta(0)=\lim _{\Gamma_{l}^{-} \ni z \rightarrow 0} \vartheta(z)$. For $l \in(0,2]$ from $(2.2)$ we have

$$
\vartheta(z)=\vartheta\left(\varrho_{l}^{-}(\theta) e^{i \theta}\right)=\theta-\arccos (l / 2)
$$

for $\theta \in(-\pi / 2+\arccos (l / 2), 0)$. Moreover $\vartheta(1)=-\arccos (l / 2)$ and $\vartheta(0)=-\pi / 2$.

Therefore we have 
ObServation 2.1. (1) If $z$ moves along $\Gamma_{k}^{+}, k \in(0,2]$, from 1 to 0 , then $\varkappa(z)$ strictly increases from $\varkappa(1)=\arccos (k / 2)$ to $\varkappa(0)=\pi / 2$. For all $z \in \Gamma_{0}^{+}, \varkappa(z)=\pi / 2$.

(2) If $z$ moves along $\Gamma_{l}^{-}, l \in(0,2]$, from 0 to 1 , then $\vartheta(z)$ strictly increases from $\vartheta(0)=-\pi / 2$ to $\vartheta(1)=-\arccos (l / 2)$. For all $z \in \Gamma_{0}^{-}$, $\vartheta(z)=-\pi / 2$.

2.3. For $x \in[0,1]$ let $k(x)=2 \cos (x \pi / 2)$. Let now $\alpha, \beta \in[0,1]$. Then $E_{k(\alpha), k(\beta)}$ is the closed set bounded by two $\operatorname{arcs} \Gamma_{k(\alpha)}^{+}$and $\Gamma_{k(\beta)}^{-}$which make an angle of $\pi(1-(\alpha+\beta) / 2)$ at their points of intersection, i.e. at 0 and 1 . Note that $E_{k(\alpha), k(\alpha)}, \alpha \in(0,1)$, is a lens-shape set bounded by two symmetrical circular $\operatorname{arcs} \Gamma_{k(\alpha)}^{+}$and $\Gamma_{k(\alpha)}^{-}$which intersect at an angle of $\pi(1-\alpha)$. Clearly, $E_{k(1), k(1)}=[0,1]$ and $E_{k(0), k(0)}=\overline{\mathbb{D}(1 / 2,1 / 2)}$.

Let $\alpha, \beta \in[0,1)$. By $(2.1)$ and $(2.2)$ with $k=k(\alpha)=2 \cos (\alpha \pi / 2) \in(0,2]$ and $l=k(\beta)=2 \cos (\beta \pi / 2) \in(0,2], \Gamma_{k(\alpha)}^{+} \backslash\{0\}$ and $\Gamma_{k(\beta)}^{-} \backslash\{0\}$ have the following polar equations:

$$
\begin{array}{r}
\Gamma_{k(\alpha)}^{+} \backslash\{0\}: \quad z=\varrho_{k(\alpha)}^{+}(\theta) e^{i \theta}=\frac{\cos (\theta+\alpha \pi / 2)}{\cos (\alpha \pi / 2)} e^{i \theta} \\
\theta \in[0,(1-\alpha) \pi / 2),
\end{array}
$$

and

$$
\begin{array}{r}
\Gamma_{k(\beta)}^{-} \backslash\{0\}: \quad z=\varrho_{k(\beta)}^{-}(\theta) e^{i \theta}=\frac{\cos (\theta-\beta \pi / 2)}{\cos (\beta \pi / 2)} e^{i \theta}, \\
\theta \in(-(1-\beta) \pi / 2,0] .
\end{array}
$$

Clearly,

$$
\begin{aligned}
\left.\varrho_{k(\alpha)}^{+}((1-\alpha) \pi / 2)\right) & =\lim _{\theta \rightarrow[(1-\alpha) \pi / 2]^{-}} \varrho_{k(\alpha)}^{+}(\theta)=0, \\
\left.\varrho_{k(\beta)}^{-}(-(1-\beta) \pi / 2)\right) & =\lim _{\theta \rightarrow[-(1-\beta) \pi / 2]^{+}} \varrho_{k(\beta)}^{-}(\theta)=0 .
\end{aligned}
$$

Now we define the class $\mathcal{Z}^{*}(\alpha, \beta)$ of strongly starlike domains of order $(\alpha, \beta)$.

Definition 2.1. A domain $\Omega \subset \mathbb{C}, \Omega \neq \mathbb{C}$, belongs to $\mathcal{Z}^{*}(\alpha, \beta), \alpha, \beta \in$ $[0,1]$, and is called strongly starlike of order $(\alpha, \beta)$ if $w E_{k(\alpha), k(\beta)} \subset \Omega$ for every $w \in \Omega$.

Strongly starlike domains of order $(\alpha, \alpha)$ will be called strongly starlike of order $\alpha$ and the set $\mathcal{Z}^{*}(\alpha, \alpha)$ will be denoted by $\mathcal{Z}^{*}(\alpha)$.

Let, for short, $\mathcal{Z}^{*}=\mathcal{Z}^{*}(1)$ denote the class of starlike domains.

REMARK 2.1. Since $\mathcal{Z}^{*}(\alpha, \beta) \subset \mathcal{Z}^{*}$ for all $\alpha, \beta \in[0,1]$, every domain in $\mathcal{Z}^{*}(\alpha, \beta)$ is simply connected.

The following lemma is obvious. 
LEMMA 2.1. $\mathcal{Z}^{*}\left(\alpha_{1}, \beta_{1}\right) \subset \mathcal{Z}^{*}\left(\alpha_{2}, \beta_{2}\right)$ for any $0 \leq \alpha_{1} \leq \alpha_{2} \leq 1$ and $0 \leq \beta_{1} \leq \beta_{2} \leq 1$

Now we prove that the classes $\mathcal{Z}^{*}(\alpha, 0)$ and $\mathcal{Z}^{*}(0, \beta)$ contain only disks centered at the origin.

Lemma 2.2. For every $\alpha, \beta \in[0,1]$,

$$
\mathcal{Z}^{*}(\alpha, 0)=\mathcal{Z}^{*}(0, \beta)=\left\{\mathbb{D}_{r}: r>0\right\} .
$$

Proof. Since, by Lemma $2.1, \mathcal{Z}^{*}(\alpha, 0) \subset \mathcal{Z}^{*}(1,0)$ for $\alpha \in[0,1]$, it is enough to prove that $\mathcal{Z}^{*}(1,0)$ consists of disks centered at the origin.

Let $\Omega \in \mathcal{Z}^{*}(1,0)$. Then $w E_{k(1), k(0)}=w E_{0,2} \subset \Omega$ for every $w \in \Omega$. Clearly, $w E_{0,2}$ is a half-disk with the boundary

$$
\partial\left(w E_{0,2}\right)=[0, w] \cup w \Gamma_{2}^{-} .
$$

Now fix $w \in \Omega$. Without loss of generality we can assume that $w=r \in \mathbb{R}_{+}$. For $n \in \mathbb{N}$ let $\delta_{n}=2 \pi / n$. Fix $n \geq 5$. Since $r E_{0,2} \subset \Omega$, we see that $w_{1}=$ $e^{-i \delta_{n}} r \cos \delta_{n} \in r \Gamma_{2}^{-}$, i.e. $w_{1} \in \Omega$. Thus $w_{1} E_{0,2} \subset \Omega$. Hence

$$
w_{2}=e^{-2 i \delta_{n}}\left|w_{1}\right| \cos \delta_{n}=e^{-2 i \delta_{n}} r \cos ^{2} \delta_{n} \in w_{1} \Gamma_{2}^{-},
$$

i.e. $w_{2} \in \Omega$. In this way we can find $n+1$ points $w_{0}, w_{1}, w_{2}, \ldots, w_{n}$ of $\Omega$ such that $w_{0}=r$ and

$$
w_{k}=e^{-i k \delta_{n}}\left|w_{k-1}\right| \cos \delta_{n}=e^{-i k \delta_{n}} r \cos ^{k} \delta_{n} \in w_{k-1} \Gamma_{2}^{-}, \quad k=1, \ldots, n .
$$

Since, in particular,

$$
w_{n}=e^{-i n \delta_{n}} r \cos ^{n} \delta_{n}=r \cos ^{n}\left(\frac{2 \pi}{n}\right),
$$

we have

$$
\lim _{n \rightarrow \infty} w_{n}=r \lim _{n \rightarrow \infty} \cos ^{n}\left(\frac{2 \pi}{n}\right)=r .
$$

Hence we deduce that $\mathbb{D}_{r} \subset \Omega$. Thus we proved that $\mathbb{D}_{|w|} \subset \Omega$ for every $w \in \Omega$, which means that $\Omega$ is a disk centered at the origin.

The proof for the class $\mathcal{Z}^{*}(0, \beta), \beta \in[0,1]$, is identical.

\section{An analytic characterization of strongly starlike functions of order $(\alpha, \beta)$}

3.1. Let $\mathcal{S}^{*}(\alpha, \beta) \subset \mathcal{S}, \alpha, \beta \in[0,1]$, denote the class of functions $f \in \mathcal{S}$ such that $f(\mathbb{D}) \in \mathcal{Z}^{*}(\alpha, \beta)$, called strongly starlike of order $(\alpha, \beta)$. For $\xi \in \mathbb{D}$ let $\mathcal{S}^{*}(\alpha, \beta ; \xi)=\mathcal{S}^{*}(\alpha, \beta) \cap \mathcal{S}(\xi)$. For $\alpha \in[0,1]$, let $\mathcal{S}^{*}(\alpha)$ denote the class $\mathcal{S}^{*}(\alpha, \alpha)$ of functions called strongly starlike of order $\alpha$ and let $\mathcal{S}^{*}(\alpha ; \xi)=$ $\mathcal{S}^{*}(\alpha, \alpha ; \xi)$ for $\xi \in \mathbb{D}$.

Observe first that by Lemma 2.2 we have 
Corollary 3.1. For any $\alpha, \beta \in[0,1]$ and every $\xi \in \mathbb{D}$,

$$
\mathcal{S}^{*}(0, \beta ; \xi)=\mathcal{S}^{*}(\alpha, 0 ; \xi)=\left\{a \varphi_{\xi}: a \in \mathbb{C} \backslash\{0\}\right\} .
$$

For this reason in the following considerations we assume that $\alpha, \beta \in$ $(0,1]$.

3.2. In the proof of Theorem 3.2 we make essential use of the fact that every $f \in \mathcal{S}^{*}(\alpha, \beta ; \xi)$ preserves strong starlikeness of order $(\alpha, \beta)$ on each hyperbolic disk centered at $\xi$, i.e. we use the following theorem proved in [L] for $\alpha=\beta \in[0,1]$ and in [LL] for $\alpha, \beta \in[0,1]$.

Theorem 3.1 ([LL]). Let $\alpha, \beta \in(0,1], \xi \in \mathbb{D}$ and $f \in \mathcal{S}(\xi)$. Then $f \in \mathcal{S}^{*}(\alpha, \beta ; \xi)$ if and only if $f(\mathbb{B}(\xi, \rho)) \in \mathcal{Z}^{*}(\alpha, \beta)$ for every $\rho>0$.

Now we reprove the analytic characterization of functions in $\mathcal{S}^{*}(\alpha, \beta ; \xi)$ first given in [LL]. The case $\alpha=\beta$ was shown in [L]. One of the goals of this paper is to present a new analytical proof of the theorem below.

Theorem 3.2. Let $\alpha, \beta \in(0,1]$ and $\xi \in \mathbb{D}$. If $f \in \mathcal{S}^{*}(\alpha, \beta ; \xi)$, then

$$
-\beta \frac{\pi}{2}<\arg \left\{\frac{(1-\bar{\xi} z)(z-\xi) f^{\prime}(z)}{f(z)}\right\}<\alpha \frac{\pi}{2}, \quad z \in \mathbb{D} .
$$

Conversely, if $f \in \mathcal{A}$ with $f^{\prime}(\xi) \neq 0$ satisfies (3.1), then $f \in \mathcal{S}^{*}(\alpha, \beta ; \xi)$.

Proof. Necessity. Let $f \in \mathcal{S}^{*}(\alpha, \beta ; \xi)$. Thus $f \in \mathcal{S}(\xi)$ and $\Omega=f(\mathbb{D}) \in$ $\mathcal{Z}^{*}(\alpha, \beta)$.

1. As $f(\xi)=0$ and $f^{\prime}(\xi) \neq 0$, the function

$$
S(z ; \xi)=\frac{(1-\bar{\xi} z)(z-\xi) f^{\prime}(z)}{f(z)}, \quad z \in \mathbb{D} \backslash\{\xi\},
$$

has a removable singularity at $z=\xi$ with

$$
S(\xi ; \xi)=\lim _{z \rightarrow \xi} \frac{(1-\bar{\xi} z)(z-\xi) f^{\prime}(z)}{f(z)}=1-|\xi|^{2} .
$$

Hence (3.1) holds for $z=\xi$.

2. Fix $z \in \mathbb{D}, z \neq \xi$. Then $z \in C_{\rho}$ for some $\rho>0$. By Theorem 3.1, $f(\mathbb{B}(\xi, \rho)) \in \mathcal{Z}^{*}(\alpha, \beta)$. Hence

$$
f(z) E_{k(\alpha), k(\beta)} \subset \overline{f(\mathbb{B}(\xi, \rho))} .
$$

Indeed, let $w_{0} \in f(z) E_{k(\alpha), k(\beta)}$, i.e. $w_{0}=f(z) u_{0}$ for some $u_{0} \in E_{k(\alpha), k(\beta)}$. Since $f(z) \in \partial f(\mathbb{B}(\xi, \rho))=f\left(C_{\rho}\right)$, there exists a sequence $\left(w_{n}\right)$ in $f(\mathbb{B}(\xi, \rho))$ convergent to $f(z)$. The inclusion $w_{n} E_{k(\alpha), k(\beta)} \subset f(\mathbb{B}(\xi, \rho))$ shows that $w_{n} u_{0} \in f(\mathbb{B}(\xi, \rho))$ for every $n \in \mathbb{N}$. Since $\lim _{n \rightarrow \infty}\left(w_{n} u_{0}\right)=f(z) u_{0}=w_{0}$, our claim is proved. 
(a) Fix $\delta \in[\alpha, 1)$. We see from (3.3) that $f(z) \varrho_{k(\delta)}^{+}(\theta) e^{i \theta} \in \overline{f(\mathbb{B}(\xi, \rho))}$ for $\theta \in[0,(1-\delta) \pi / 2)$. Hence

$$
\omega_{\theta}^{+}(\delta ; z)=f^{-1}\left(f(z) \varrho_{k(\delta)}^{+}(\theta) e^{i \theta}\right) \in \overline{\mathbb{B}(\xi, \rho)},
$$

and, consequently,

$$
\varphi_{\xi} \circ \omega_{\theta}^{+}(\delta ; z) \in \varphi_{\xi}(\overline{\mathbb{B}(\xi, \rho)})=\overline{\mathbb{D}}_{r}
$$

for $r=\tanh \rho$ and $\theta \in[0,(1-\delta) \pi / 2)$. Thus

(3.4) $\left|\varphi_{\xi} \circ \omega_{\theta}^{+}(\delta ; z)\right| \leq\left|\varphi_{\xi} \circ \omega_{0}^{+}(\delta ; z)\right|=\left|\varphi_{\xi}(z)\right|, \quad \theta \in[0,(1-\delta) \pi / 2)$.

For $\theta \in[0,(1-\delta) \pi / 2)$ let

$$
\psi_{z}^{+}(\delta ; \theta)=\varphi_{\xi} \circ \omega_{\theta}^{+}(\delta ; z) .
$$

Since $\left|\psi_{z}^{+}(\delta ; 0)\right|=\left|\varphi_{\xi}(z)\right|$ and $\psi_{z}^{+}(\delta ; \theta) \neq 0$ for $\theta \in[0,(1-\delta) \pi / 2)$, from (3.4) we have

$$
\begin{aligned}
0 & \lim _{\theta \rightarrow 0^{+}} \frac{\left|\psi_{z}^{+}(\delta ; \theta)\right|-\left|\psi_{z}^{+}(\delta ; 0)\right|}{\theta}=\frac{\partial}{\partial \theta}\left|\psi_{z}^{+}(\delta ; \theta)\right|_{\mid \theta=0} \\
& =\frac{\partial}{\partial \theta}\left|\varphi_{\xi} \circ \omega_{\theta}^{+}(\delta ; z)\right|_{\mid \theta=0}=\left|\varphi_{\xi} \circ \omega_{\theta}^{+}(\delta ; z)\right|_{\mid \theta=0} \operatorname{Re}\left\{\frac{\frac{\partial}{\partial \theta} \varphi_{\xi} \circ \omega_{\theta}^{+}(\delta ; z)}{\varphi_{\xi} \circ \omega_{\theta}^{+}(\delta ; z)}\right\}_{\mid \theta=0} \\
& =\left|\varphi_{\xi}(z)\right| \operatorname{Re}\left\{\frac{\varphi_{\xi}^{\prime}\left(\omega_{\theta}^{+}(\delta ; z)\right) \frac{\partial}{\partial \theta} \omega_{\theta}^{+}(\delta ; z)}{\varphi_{\xi}(z)}\right\}_{\mid \theta=0} \\
& =\left|\varphi_{\xi}(z)\right| \operatorname{Re}\left\{\frac{1-|\xi|^{2}}{\left(1-\bar{\xi} \omega_{\theta}^{+}(\delta ; z)\right)^{2} \varphi_{\xi}(z)} \frac{\partial}{\partial \theta} f^{-1}\left(f(z) \varrho_{k(\delta)}^{+}(\theta) e^{i \theta}\right)\right\}_{\mid \theta=0} \\
& =\left|\varphi_{\xi}(z)\right| \operatorname{Re}\left\{\frac{1-|\xi|^{2}}{(1-\bar{\xi} z)(z-\xi)} \frac{f(z)\left(\left(\varrho_{k(\delta)}^{+}\right)^{\prime}(\theta) e^{i \theta}+i \varrho_{k(\delta)}^{+}(\theta) e^{i \theta}\right)}{f^{\prime}\left(f-1\left(f(z) \varrho_{k(\delta)}^{+}(\theta) e^{i \theta}\right)\right)}\right\}_{\mid \theta=0} \\
& =\left(1-|\xi|^{2}\right)\left|\varphi_{\xi}(z)\right| \operatorname{Re}\left\{(-\tan (\delta \pi / 2)+i) \frac{f(z)}{(1-\bar{\xi} z)(z-\xi) f^{\prime}(z)}\right\} \\
& =\frac{\left(1-|\xi|^{2}\right)\left|\varphi_{\xi}(z)\right|}{\cos (\delta \pi / 2)} \operatorname{Re}\left\{i e^{i \delta \pi / 2} \frac{f(z)}{(1-\bar{\xi} z)(z-\xi) f^{\prime}(z)}\right\}, \quad z \in \mathbb{D} \backslash\{\xi\} .
\end{aligned}
$$

Thus

$$
0 \leq \arg \left\{e^{i \delta \pi / 2} \frac{f(z)}{(1-\bar{\xi} z)(z-\xi) f^{\prime}(z)}\right\} \leq \pi, \quad z \in \mathbb{D} \backslash\{\xi\} .
$$

Hence

$$
-\pi+\delta \frac{\pi}{2} \leq \arg \left\{\frac{(1-\bar{\xi} z)(z-\xi) f^{\prime}(z)}{f(z)}\right\} \leq \delta \frac{\pi}{2}, \quad z \in \mathbb{D} \backslash\{\xi\} .
$$


Since the last inequalities are true for every $\delta \in[\alpha, 1)$, we see that

$$
-\frac{\pi}{2} \leq \arg \left\{\frac{(1-\bar{\xi} z)(z-\xi) f^{\prime}(z)}{f(z)}\right\} \leq \alpha \frac{\pi}{2}, \quad z \in \mathbb{D} \backslash\{\xi\} .
$$

(b) Fix $\delta \in[\beta, 1)$. We see from (3.3) that $f(z) \varrho_{k(\delta)}^{-}(\theta) e^{i \theta} \in \overline{f(\mathbb{B}(\xi, \rho))}$ for $\theta \in(-(1-\delta) \pi / 2,0]$. Hence

$$
\omega_{\theta}^{-}(\delta ; z)=f^{-1}\left(f(z) \varrho_{k(\delta)}^{-}(\theta) e^{i \theta}\right) \in \overline{\mathbb{B}(\xi, \rho)},
$$

and, consequently,

$$
\varphi_{\xi} \circ \omega_{\theta}^{-}(\delta ; z) \in \varphi_{\xi}(\overline{\mathbb{B}(\xi, \rho)})=\overline{\mathbb{D}}_{r}
$$

for $r=\tanh \rho$ and $\theta \in(-(1-\delta) \pi / 2,0]$. Thus

(3.6) $\left|\varphi_{\xi} \circ \omega_{\theta}^{-}(\delta ; z)\right| \leq\left|\varphi_{\xi} \circ \omega_{0}^{-}(\delta ; z)\right|=\left|\varphi_{\xi}(z)\right|, \quad \theta \in(-(1-\delta) \pi / 2,0]$.

For $\theta \in(-(1-\delta) \pi / 2,0]$ let

$$
\psi_{z}^{-}(\delta ; \theta)=\varphi_{\xi} \circ \omega_{\theta}^{-}(\delta ; z) .
$$

Since $\left|\psi_{z}^{-}(\delta ; 0)\right|=\left|\varphi_{\xi}(z)\right|$ and $\psi_{z}^{-}(\delta ; \theta) \neq 0$ for $\theta \in(-(1-\delta) \pi / 2,0]$, calculating as in part $2(\mathrm{a})$ of this proof, from (3.6) we have

$$
\begin{aligned}
0 & \leq \lim _{\theta \rightarrow 0^{-}} \frac{\left|\psi_{z}^{-}(\delta ; \theta)\right|-\left|\psi_{z}^{-}(\delta ; 0)\right|}{\theta}=\frac{\partial}{\partial \theta}\left|\psi_{z}^{-}(\delta ; \theta)\right|_{\mid \theta=0} \\
& =\frac{\left(1-|\xi|^{2}\right)\left|\varphi_{\xi}(z)\right|}{\cos (\delta \pi / 2)} \operatorname{Re}\left\{i e^{-i \delta \pi / 2} \frac{f(z)}{(1-\bar{\xi} z)(z-\xi) f^{\prime}(z)}\right\}, \quad z \in \mathbb{D} \backslash\{\xi\} .
\end{aligned}
$$

Thus

$$
-\pi \leq \arg \left\{e^{-i \delta \pi / 2} \frac{f(z)}{(1-\bar{\xi} z)(z-\xi) f^{\prime}(z)}\right\} \leq 0, \quad z \in \mathbb{D} \backslash\{\xi\} .
$$

Hence

$$
-\delta \frac{\pi}{2} \leq \arg \left\{\frac{(1-\bar{\xi} z)(z-\xi) f^{\prime}(z)}{f(z)}\right\} \leq \pi-\delta \frac{\pi}{2}, \quad z \in \mathbb{D} \backslash\{\xi\} .
$$

Since the last inequalities are true for every $\delta \in[\beta, 1)$, we see that

$$
-\beta \frac{\pi}{2} \leq \arg \left\{\frac{(1-\bar{\xi} z)(z-\xi) f^{\prime}(z)}{f(z)}\right\} \leq \frac{\pi}{2}, \quad z \in \mathbb{D} \backslash\{\xi\} .
$$

(c) From (3.3) we see that $t f(z) \in \overline{f(\mathbb{B}(\xi, \rho))}$ for every $t \in[0,1]$. Hence

$$
\omega_{t}(z)=f^{-1}(t f(z)) \in \overline{\mathbb{B}(\xi, \rho)},
$$

and, consequently,

$$
\varphi_{\xi} \circ \omega_{t}(z) \in \varphi_{\xi}(\overline{\mathbb{B}(\xi, \rho)})=\overline{\mathbb{D}}_{r}
$$

for $r=\tanh \rho$ and $t \in[0,1]$. Thus

$$
\left|\varphi_{\xi} \circ \omega_{t}(z)\right| \leq\left|\varphi_{\xi} \circ \omega_{1}(z)\right|=\left|\varphi_{\xi}(z)\right|, \quad t \in[0,1] .
$$


For $t \in[0,1]$ let

$$
\psi_{z}(t)=\varphi_{\xi} \circ \omega_{t}(z) .
$$

Since $\left|\psi_{z}(1)\right|=\left|\varphi_{\xi}(z)\right|$ and $\psi_{z}(t) \neq 0$ for $t \in(0,1]$, calculating as in parts 2(a)-2(b) of this proof, from (3.8) we have

$$
\begin{aligned}
0 & \leq \lim _{t \rightarrow 1^{-}} \frac{\left|\psi_{z}(t)\right|-\left|\psi_{z}(1)\right|}{t-1}=\frac{\partial}{\partial t}\left|\psi_{z}(t)\right|_{\mid t=1} \\
& =\left(1-|\xi|^{2}\right)\left|\varphi_{\xi}(z)\right| \operatorname{Re}\left\{\frac{f(z)}{(1-\bar{\xi} z)(z-\xi) f^{\prime}(z)}\right\}, \quad z \in \mathbb{D} \backslash\{\xi\} .
\end{aligned}
$$

Thus we proved that

$$
\operatorname{Re}\left\{\frac{(1-\bar{\xi} z)(z-\xi) f^{\prime}(z)}{f(z)}\right\} \geq 0, \quad z \in \mathbb{D} \backslash\{\xi\} .
$$

3. Finally, from part 1 of this proof, (3.5), (3.7) and (3.9) it follows that every $f \in \mathcal{S}^{*}(\alpha, \beta ; \xi)$ satisfies the condition

$$
-\beta \frac{\pi}{2} \leq \arg \left\{\frac{(1-\bar{\xi} z)(z-\xi) f^{\prime}(z)}{f(z)}\right\} \leq \alpha \frac{\pi}{2}, \quad z \in \mathbb{D} .
$$

Observe that (3.10) follows from parts 2(a) and 2(b). Part 2(c) shows that (3.10) remains valid in the case $\delta=1$.

4. Suppose that the right inequality in (3.10) is equality for some point of $\mathbb{D}$. Then by the maximum principle for harmonic functions it holds for every point in $\mathbb{D}$. But this is impossible since $S(\xi ; \xi)$ is a real number. Therefore the right inequality of (3.10) is strict, and similarly for the left inequality.

Sufficiency. Assume that $f \in \mathcal{A}$ with $f^{\prime}(\xi) \neq 0$ satisfies (3.1). We will prove that $f \in \mathcal{S}^{*}(\alpha, \beta ; \xi)$.

A. We first prove that $f$ is univalent in $\mathbb{D}$.

1. Since $f$ satisfies $(3.1)$ and $f^{\prime}(\xi) \neq 0$, we see that $S$ as a function of $z$ has no pole and no zero in $\mathbb{D}$, which yields $f(\xi)=0$. In consequence, $f \in \mathcal{A}(\xi)$.

By assumption we have $f^{\prime}(\xi) \neq 0$. Suppose now that $f^{\prime}\left(z_{0}\right)=0$ for some $z_{0} \in \mathbb{D} \backslash\{\xi\}$. In view of $(3.1)$ we must have $f\left(z_{0}\right)=0$. Then

$$
f(z)=\left(z-z_{0}\right)^{m} g(z), \quad z \in \mathbb{D},
$$

where $g \in \mathcal{A}$ with $g\left(z_{0}\right) \neq 0$ and $m \geq 2$. Hence

$$
\frac{f^{\prime}(z)}{f(z)}=\frac{m}{z-z_{0}}+\frac{g^{\prime}(z)}{g(z)}, \quad z \in \mathbb{D} .
$$

Thus $S$ has a simple pole at $z_{0}$, which is impossible since $S$ is analytic in $\mathbb{D}$ as a function of $z$. Consequently, $f^{\prime}$ is nonvanishing in $\mathbb{D}$. This together with (3.1) implies at once that $f$ is nonvanishing in $\mathbb{D} \backslash\{\xi\}$. Since $f^{\prime}(\xi) \neq 0$, 
this shows that $\xi$ is a unique single zero of $f$ in $\mathbb{D}$. Fix $\rho>0$. Using the principle of the argument we conclude that

$$
\Delta_{C_{\rho}} \arg f(z)=2 \pi n\left(0, \Gamma_{\rho}\right)=2 \pi,
$$

where $n\left(0, \Gamma_{\rho}\right)$ denotes the index (winding number) of $\Gamma_{\rho}$ with respect to the origin. This means that $\Gamma_{\rho}$ surrounds the origin exactly once.

2. Using the parametrization (1.1) of $C_{\rho}, \rho>0$, we get

$$
\begin{aligned}
& c_{\rho}^{\prime}(\theta)=\frac{i\left(1-|\xi|^{2}\right) R e^{i \theta}}{\left(1+\bar{\xi} R e^{i \theta}\right)^{2}}=i \frac{1-|\xi|^{2}}{1+\bar{\xi} R e^{i \theta}} \cdot \frac{R e^{i \theta}}{1+\bar{\xi} R e^{i \theta}} \\
& =\frac{i}{1-|\xi|^{2}}\left(1-\bar{\xi} \frac{R e^{i \theta}+\xi}{1+\bar{\xi} R e^{i \theta}}\right) \frac{R e^{i \theta}-|\xi|^{2} R e^{i \theta}}{1+\bar{\xi} R e^{i \theta}}=\frac{i(1-\bar{\xi} z)(z-\xi)}{1-|\xi|^{2}} .
\end{aligned}
$$

Since $f$ is locally univalent in $\mathbb{D}$ and nonvanishing in $\mathbb{D} \backslash\{\xi\}$, we see that $\arg f(z)$ is well defined locally on each circle $C_{\rho}, \rho>0$. Moreover, from (3.11) and (3.1) for every $\rho>0$ we have

$$
\begin{aligned}
\frac{d}{d \theta} \arg f\left(c_{\rho}(\theta)\right) & =\frac{d}{d \theta} \operatorname{Re} \log f\left(c_{\rho}(\theta)\right)=\operatorname{Re}\left\{\frac{c_{\rho}^{\prime}(\theta) f^{\prime}\left(c_{\rho}(\theta)\right)}{f\left(c_{\rho}(\theta)\right)}\right\} \\
& =\operatorname{Re}\left\{\frac{(1-\bar{\xi} z)(z-\xi) f^{\prime}(z)}{f(z)}\right\}>0, \quad z \in \mathbb{D} \backslash\{\xi\},
\end{aligned}
$$

where $z=c_{\rho}(\theta), \theta \in[0,2 \pi)$. Thus for every $\rho>0$ the function

$$
[0,2 \pi) \ni \theta \mapsto \arg f\left(c_{\rho}(\theta)\right)
$$

is increasing. Therefore $\Gamma_{\rho}$ has no multiple points. This and part 1 of this proof show that $f$ is injective on $C_{\rho}$. From [P1, Corollary 9.5] it follows that $f$ is univalent in $\mathbb{B}(\xi, \rho)$. Since $\rho>0$ was arbitrary, this yields the univalence of $f$ in $\mathbb{D}$.

B. Now we prove that $f(\mathbb{D}) \in \mathcal{Z}^{*}(\alpha, \beta)$.

1. It was observed in part $\mathrm{A} 1$ of this proof that $f(\xi)=0$, so $0 \in f(\mathbb{D})$.

2. Fix $z \in \mathbb{D}, z \neq \xi$. Then $z \in C_{\rho}$ for some $\rho>0$.

(a) Fix $\delta \in[\alpha, 1)$. Set

$$
\theta_{z}^{+}(\delta)=\sup \left\{\theta \in[0,(1-\delta) \pi / 2):\left\{f(z) \varrho_{k(\delta)}^{+}(t) e^{i t}: t \in[0, \theta)\right\} \subset f(\mathbb{D})\right\} .
$$

Since $f$ is an open mapping, $\theta_{z}^{+}(\delta)>0$. For $\theta \in\left[0, \theta_{z}^{+}(\delta)\right)$ define

$$
\omega_{\theta}^{+}(\delta ; z)=f^{-1}\left(f(z) \varrho_{k(\delta)}^{+}(\theta) e^{i \theta}\right), \quad \psi_{z}^{+}(\delta ; \theta)=\varphi_{\xi} \circ \omega_{\theta}^{+}(\delta ; z) .
$$


For $\theta \in\left[0, \theta_{z}^{+}(\delta)\right)$ we have

$$
\begin{aligned}
& \frac{\partial}{\partial \theta}\left|\psi_{z}^{+}(\delta ; \theta)\right|=\frac{\partial}{\partial \theta}\left|\varphi_{\xi} \circ \omega_{\theta}^{+}(\delta ; z)\right| \\
& =\left|\varphi_{\xi}\left(\omega_{\theta}^{+}(\delta ; z)\right)\right| \operatorname{Re}\left\{\frac{\frac{\partial}{\partial \theta} \varphi_{\xi} \circ \omega_{\theta}^{+}(\delta ; z)}{\varphi_{\xi}\left(\omega_{\theta}^{+}(\delta ; z)\right)}\right\} \\
& =\left|\varphi_{\xi}\left(\omega_{\theta}^{+}(\delta ; z)\right)\right| \operatorname{Re}\left\{\frac{\varphi_{\xi}^{\prime}\left(\omega_{\theta}^{+}(\delta ; z)\right) \frac{\partial}{\partial \theta} \omega_{\theta}^{+}(\delta ; z)}{\varphi_{\xi}\left(\omega_{\theta}^{+}(\delta ; z)\right)}\right\} \\
& =\left|\varphi_{\xi}\left(\omega_{\theta}^{+}(\delta ; z)\right)\right| \\
& \times \operatorname{Re}\left\{\frac{1-|\xi|^{2}}{\left(1-\bar{\xi} \omega_{\theta}^{+}(\delta ; z)\right)^{2} \varphi_{\xi}\left(\omega_{\theta}^{+}(\delta ; z)\right)} \frac{\partial}{\partial \theta} f^{-1}\left(f(z) \varrho_{k(\delta)}^{+}(\theta) e^{i \theta}\right)\right\} \\
& =\left|\varphi_{\xi}\left(\omega_{\theta}^{+}(\delta ; z)\right)\right| \operatorname{Re}\left\{\frac{\left(1-|\xi|^{2}\right) f(z)\left(\left(\varrho_{k(\delta)}^{+}\right)^{\prime}(\theta) e^{i \theta}+i \varrho_{k(\delta)}^{+}(\theta) e^{i \theta}\right)}{\left(1-\bar{\xi} \omega_{\theta}^{+}(\delta ; z)\right)\left(\omega_{\theta}^{+}(\delta ; z)-\xi\right) f^{\prime}\left(\omega_{\theta}^{+}(\delta ; z)\right)}\right\} \\
& =\frac{\left(1-|\xi|^{2}\right)\left|\varphi_{\xi}\left(\omega_{\theta}^{+}(\delta ; z)\right)\right|}{\cos (\delta \pi / 2)} \operatorname{Re}\left\{\frac{i e^{i(\theta+\delta \pi / 2)} e^{i \theta} f(z)}{\left(1-\bar{\xi} \omega_{\theta}^{+}(\delta ; z)\right)\left(\omega_{\theta}^{+}(\delta ; z)-\xi\right) f^{\prime}\left(\omega_{\theta}^{+}(\delta ; z)\right)}\right\} \\
& =\frac{\left(1-|\xi|^{2}\right)\left|\varphi_{\xi}\left(\omega_{\theta}^{+}(\delta ; z)\right)\right|}{\varrho_{k(\delta)}^{+}(\theta) \cos (\delta \pi / 2)} \operatorname{Re}\left\{\frac{i e^{i(\theta+\delta \pi / 2)} f\left(\omega_{\theta}^{+}(\delta ; z)\right)}{\left(1-\bar{\xi} \omega_{\theta}^{+}(\delta ; z)\right)\left(\omega_{\theta}^{+}(\delta ; z)-\xi\right) f^{\prime}\left(\omega_{\theta}^{+}(\delta ; z)\right)}\right\} \\
& =\frac{\left(1-|\xi|^{2}\right)\left|\varphi_{\xi}(u)\right|}{\varrho_{k(\delta)}^{+}(\theta) \cos (\delta \pi / 2)} \operatorname{Re}\left\{\frac{i e^{i(\theta+\delta \pi / 2)} f(u)}{(1-\bar{\xi} u)(u-\xi) f^{\prime}(u)}\right\},
\end{aligned}
$$

where $u=\omega_{\theta}^{+}(\delta ; z)$. Observe that

$$
\frac{\pi}{2}<\arg \left\{\frac{i e^{i(\theta+\delta \pi / 2)} f(u)}{(1-\bar{\xi} u)(u-\xi) f^{\prime}(u)}\right\}<\frac{3}{2} \pi
$$

if and only if

$$
-\pi+\theta+\delta \frac{\pi}{2}<\arg \left\{\frac{(1-\bar{\xi} u)(u-\xi) f^{\prime}(u)}{f(u)}\right\}<\theta+\delta \frac{\pi}{2} .
$$

Since for $\alpha \leq \delta<1$ and $0 \leq \theta<\theta_{z}^{+}(\delta) \leq(1-\delta) \pi / 2$ we have

$$
-\pi+\theta+\delta \frac{\pi}{2} \leq-\frac{\pi}{2}, \quad \theta+\delta \frac{\pi}{2} \geq \alpha \frac{\pi}{2},
$$

(3.14) follows from (3.1). This implies that the function

$$
\left[0, \theta_{z}^{+}(\delta)\right) \ni \theta \mapsto\left|\psi_{z}^{+}(\delta ; \theta)\right|
$$

is strictly decreasing for every $\delta \in[\alpha, 1)$, so $\left|\psi_{z}^{+}(\delta ; \theta)\right|<\left|\psi_{z}^{+}(\delta ; 0)\right|=\left|\varphi_{\xi}(z)\right|$ 
and, consequently,

$$
\varphi_{-\xi}\left(\psi_{z}^{+}(\delta ; \theta)\right)=f^{-1}\left(f(z) \varrho_{k(\delta)}^{+}(\theta) e^{i \theta}\right) \in \mathbb{B}(\xi, \rho)
$$

for $\theta \in\left(0, \theta_{z}^{+}(\delta)\right)$. Thus

$$
f\left(\varphi_{-\xi}\left(\psi_{z}^{+}(\delta ; \theta)\right)\right)=f(z) \varrho_{k(\delta)}^{+}(\theta) e^{i \theta} \in f(\mathbb{B}(\xi, \rho)), \quad \theta \in\left(0, \theta_{z}^{+}(\delta)\right),
$$

i.e., for every $z \in C_{\rho}$,

$$
\left\{f(z) \varrho_{k(\delta)}^{+}(\theta) e^{i \theta}: \theta \in\left(0, \theta_{z}^{+}(\delta)\right)\right\} \subset f(\mathbb{B}(\xi, \rho)) .
$$

Let

$$
w_{z}^{+}(\delta)= \begin{cases}f(z) \varrho_{k(\delta)}^{+}\left(\theta_{z}^{+}(\delta)\right) e^{i \theta_{z}^{+}(\delta)}, & \theta_{z}^{+}(\delta) \neq(1-\delta) \pi / 2, \\ 0, & \theta_{z}^{+}(\delta)=(1-\delta) \pi / 2 .\end{cases}
$$

Suppose that $w_{z}^{+}(\delta) \neq 0$, i.e. $\theta_{z}^{+}(\delta) \neq(1-\delta) \pi / 2$. By (3.15), we have $w_{z}^{+}(\delta) \in \partial f(\mathbb{B}(\xi, \rho))=f\left(C_{\rho}\right)$. Consequently, there exists $z^{+}(\delta) \in C_{\rho}$ such that $w_{z}^{+}(\delta)=f\left(z^{+}(\delta)\right)$. Hence $\mathbb{D}\left(w_{z}^{+}(\delta), \varepsilon\right) \subset f(\mathbb{D})$ for some $\varepsilon>0$. Thus we conclude that

$$
\left\{f(z) \varrho_{k(\delta)}^{+}(\theta) e^{i \theta}: \theta \in\left[\theta_{z}^{+}(\delta), \theta^{+}(\varepsilon)\right)\right\} \subset f(\mathbb{D})
$$

for some $\theta^{+}(\varepsilon)>\theta_{z}^{+}(\delta)$, so

$$
\left\{f(z) \varrho_{k(\delta)}^{+}(\theta) e^{i \theta}: \theta \in\left[0, \theta^{+}(\varepsilon)\right)\right\} \subset f(\mathbb{D}) .
$$

This contradicts the definition of $\theta_{z}^{+}(\delta)$ and shows that $\theta_{z}^{+}(\delta)=(1-\delta) \pi / 2$ and $w_{z}^{+}(\delta)=0$. In this way we proved that

$$
\left\{f(z) \varrho_{k(\delta)}^{+}(\theta) e^{i \theta}: \theta \in[0,(1-\delta) \pi / 2)\right\} \subset f(\mathbb{D})
$$

for every $\delta \in[\alpha, 1)$.

(b) Fix $\delta \in[\beta, 1)$. Set

$$
\theta_{z}^{-}(\delta)=\inf \left\{\theta \in(-(1-\delta) \pi / 2,0]:\left\{f(z) \varrho_{k(\delta)}^{-}(t) e^{i t}: t \in(\theta, 0]\right\} \subset f(\mathbb{D})\right\} .
$$

Since $f$ is an open mapping, $\theta_{z}^{-}(\delta)<0$. For $\theta \in\left(\theta_{z}^{-}(\delta), 0\right]$ define

$$
\omega_{\theta}^{-}(\delta ; z)=f^{-1}\left(f(z) \varrho_{k(\delta)}^{-}(\theta) e^{i \theta}\right), \quad \psi_{z}^{-}(\delta ; \theta)=\varphi_{\xi} \circ \omega_{\theta}^{-}(\delta ; z) .
$$

As in (3.13), for $\theta \in\left(\theta_{z}^{-}(\delta), 0\right]$, we have

$$
\frac{\partial}{\partial \theta}\left|\psi_{z}^{-}(\delta ; \theta)\right|=\frac{\left(1-|\xi|^{2}\right)\left|\varphi_{\xi}(u)\right|}{\varrho_{k(\delta)}^{-}(\theta) \cos (\delta \pi / 2)} \operatorname{Re}\left\{\frac{i e^{i(\theta-\delta \pi / 2)} f(u)}{(1-\bar{\xi} u)(u-\xi) f^{\prime}(u)}\right\},
$$

where $u=\omega_{\theta}^{-}(\delta ; z)$. Observe that

$$
-\frac{\pi}{2}<\arg \left\{\frac{i e^{i(\theta-\delta \pi / 2)} f(u)}{(1-\bar{\xi} u)(u-\xi) f^{\prime}(u)}\right\}<\frac{\pi}{2}
$$


if and only if

$$
\theta-\delta \frac{\pi}{2}<\arg \left\{\frac{(1-\bar{\xi} u)(u-\xi) f^{\prime}(u)}{f(u)}\right\}<\pi+\theta-\delta \frac{\pi}{2} .
$$

Since for $\beta \leq \delta<1$ and $-(1-\delta) \pi / 2 \leq \theta_{z}^{-}(\delta)<\theta \leq 0$ we have

$$
\theta-\delta \frac{\pi}{2} \leq-\beta \frac{\pi}{2}, \quad \pi+\theta-\delta \frac{\pi}{2} \geq \frac{\pi}{2}
$$

(3.1) yields (3.17). This implies that the function

$$
\left(\theta_{z}^{-}(\delta), 0\right] \ni \theta \mapsto\left|\psi_{z}^{-}(\delta ; \theta)\right|
$$

is strictly increasing for every $\delta \in[\beta, 1)$, so $\left|\psi_{z}^{-}(\delta ; \theta)\right|<\left|\psi_{z}^{-}(\delta ; 0)\right|=\left|\varphi_{\xi}(z)\right|$ and, consequently,

$$
\varphi_{-\xi}\left(\psi_{z}^{-}(\delta ; \theta)\right)=f^{-1}\left(f(z) \varrho_{k(\delta)}^{-}(\theta) e^{i \theta}\right) \in \mathbb{B}(\xi, \rho)
$$

for $\theta \in\left(\theta_{z}^{-}(\delta), 0\right)$. Thus

$$
f\left(\varphi_{-\xi}\left(\psi_{z}^{-}(\delta ; \theta)\right)\right)=f(z) \varrho_{k(\delta)}^{-}(\theta) e^{i \theta} \in f(\mathbb{B}(\xi, \rho)), \quad \theta \in\left(\theta_{z}^{-}(\delta), 0\right),
$$

i.e., for every $z \in C_{\rho}$,

$$
\left\{f(z) \varrho_{k(\delta)}^{-}(\theta) e^{i \theta}: \theta \in\left(\theta_{z}^{-}(\delta)\right), 0\right\} \subset f(\mathbb{B}(\xi, \rho))
$$

Let

$$
w_{z}^{-}(\delta)= \begin{cases}f(z) \varrho_{k(\delta)}^{-}\left(\theta_{z}^{-}(\delta)\right) e^{i \theta_{z}^{-}(\delta)}, & \theta_{z}^{-}(\delta) \neq-(1-\delta) \pi / 2, \\ 0, & \theta_{z}^{-}(\delta)=-(1-\delta) \pi / 2 .\end{cases}
$$

Suppose that $w_{z}^{-}(\delta) \neq 0$, i.e. $\theta_{z}^{-}(\delta) \neq-(1-\delta) \pi / 2$. By $(3.18)$, we have $w_{z}^{-}(\delta) \in \partial f(\mathbb{B}(\xi, \rho))=f\left(C_{\rho}\right)$. Consequently, there exists $z^{-}(\delta) \in C_{\rho}$ such that $w_{z}^{-}(\delta)=f\left(z^{-}(\delta)\right)$. Hence $\mathbb{D}\left(w_{z}^{-}(\delta), \varepsilon\right) \subset f(\mathbb{D})$ for some $\varepsilon>0$. Thus we conclude that

$$
\left\{f(z) \varrho_{k(\delta)}^{-}(\theta) e^{i \theta}: \theta \in\left(\theta^{-}(\varepsilon), \theta_{z}^{-}(\delta)\right]\right\} \subset f(\mathbb{D})
$$

for some $\theta^{-}(\varepsilon)<\theta_{z}^{-}(\delta)$, so

$$
\left\{f(z) \varrho_{k(\delta)}^{-}(\theta) e^{i \theta}: \theta \in\left(\theta^{-}(\varepsilon), 0\right]\right\} \subset f(\mathbb{D}) .
$$

This contradicts the definition of $\theta_{z}^{-}(\delta)$ and shows $\theta_{z}^{-}(\delta)=-(1-\delta) \pi / 2$ and $w_{z}^{-}(\delta)=0$. In this way we proved that

$$
\left\{f(z) \varrho_{k(\delta)}^{-}(\theta) e^{i \theta}: \theta \in(-(1-\delta) \pi / 2,0]\right\} \subset f(\mathbb{D})
$$

for every $\delta \in[\beta, 1)$.

(c) Set

$$
t_{z}=\inf \{t \in[0,1]:[t f(z), f(z)] \subset f(\mathbb{D})\} .
$$

Since $f$ is an open mapping, $t_{z}<1$. For $t \in\left(t_{z}, 1\right]$ define

$$
\omega_{t}(z)=f^{-1}(t f(z)), \quad \psi_{z}(t)=\varphi_{\xi} \circ \omega_{t}(z) .
$$


As in (3.13) for $t \in\left(t_{z}, 1\right]$ we have

$$
\frac{d}{d t}\left|\psi_{z}(t)\right|=\frac{1}{t}\left(1-|\xi|^{2}\right)\left|\varphi_{\xi}(u)\right| \operatorname{Re}\left\{\frac{f(u)}{(1-\bar{\xi} u)(u-\xi) f^{\prime}(u)}\right\},
$$

where $u=\omega_{t}(z)$. Thus from (3.1) we obtain

$$
\frac{d}{d t}\left|\psi_{z}(t)\right|>0, \quad t \in\left(t_{z}, 1\right] .
$$

This implies that the function $\left(t_{z}, 1\right] \ni t \mapsto\left|\psi_{z}(t)\right|$ is strictly increasing, so $\left|\psi_{z}(t)\right|<\left|\psi_{z}(1)\right|=\left|\varphi_{\xi}(z)\right|$ and, consequently,

$$
\varphi_{-\xi}\left(\psi_{z}(t)\right)=f^{-1}(t f(z)) \in \mathbb{B}(\xi, \rho)
$$

for $t \in\left(t_{z}, 1\right)$. Thus

$$
f\left(\varphi_{-\xi}\left(\psi_{z}(t)\right)\right)=t f(z) \in f(\mathbb{B}(\xi, \rho)), \quad t \in\left(t_{z}, 1\right),
$$

i.e., for every $z \in C_{\rho}$,

$$
\left(w_{z}, f(z)\right) \subset f(\mathbb{B}(\xi, \rho)),
$$

where $w_{z}=t_{z} f(z)$.

Suppose that $w_{z} \neq 0$, i.e. $t_{z} \neq 0$. By (3.20), $w_{z} \in \partial f(\mathbb{B}(\xi, \rho))=f\left(C_{\rho}\right)$. Consequently, there exists $z_{0} \in C_{\rho}$ such that $w_{z}=f\left(z_{0}\right)$. Hence $\mathbb{D}\left(w_{z}, \varepsilon\right) \subset$ $f(\mathbb{D})$ for some $\varepsilon>0$. Thus we conclude that

$$
\left(t(\varepsilon) f(z), t_{z} f(z)\right] \subset f(\mathbb{D})
$$

for some $t(\varepsilon)<t_{z}$, so

$$
(t(\varepsilon) f(z), f(z)] \subset f(\mathbb{D}) .
$$

This contradicts the definition of $t_{z}$ and shows that $t_{z}=0$ and $w_{z}=0$. In this way we proved that

$$
(0, f(z)] \subset f(\mathbb{D}) .
$$

3. Finally, from part B1 of this proof, (3.16), (3.19) and (3.21) it follows that $f(\mathbb{D}) \in \mathcal{Z}^{*}(\alpha, \beta)$. This ends the proof of the sufficiency.

Corollary 3.2. Let $\alpha, \beta \in(0,1]$. If $f \in \mathcal{S}^{*}(\alpha, \beta ; 0)$, then

$$
-\beta \frac{\pi}{2}<\arg \left\{\frac{z f^{\prime}(z)}{f(z)}\right\}<\alpha \frac{\pi}{2}, \quad z \in \mathbb{D} .
$$

Conversely, if $f \in \mathcal{A}$ with $f^{\prime}(0) \neq 0$ satisfies (3.22), then $f \in \mathcal{S}^{*}(\alpha, \beta ; 0)$.

\section{Spiralshaped domains of order $(\alpha, \beta)$}

4.1. For $\alpha, \beta \in[0,1]$ we denote by $L^{+}(\alpha)$ and $L^{-}(\beta)$ the closures of the sets $\left\{\exp \left(-e^{-i(1-\alpha) \pi / 2} t\right): t \geq 0\right\}$ and $\left\{\exp \left(e^{i(1-\beta) \pi / 2} t\right): t \leq 0\right\}$, respectively.

(a) Let $\alpha, \beta \in(0,1)$. Then $L^{+}(\alpha)$ and $L^{-}(\beta)$ are two closed logarithmic spirals joining 0 and 1 parametrized as in their definitions. We see that $L^{+}(\alpha)$ 
from 1 to 0 , and $L^{-}(\beta)$ from 0 to 1 . It is convenient to write $L^{+}(\alpha) \backslash\{0\}$ and $L^{-}(\beta) \backslash\{0\}$ in polar coordinates:

$$
\begin{array}{lll}
L^{+}(\alpha) \backslash\{0\}: & z=r_{\alpha}^{+}(\theta) e^{i \theta}, & \theta \in[0, \infty), \\
L^{-}(\beta) \backslash\{0\}: & z=r_{\beta}^{-}(\theta) e^{i \theta}, & \theta \in(-\infty, 0],
\end{array}
$$

where

$$
r_{\alpha}^{+}(\theta)=\exp (-\theta \tan (\alpha \pi / 2)), \quad r_{\beta}^{-}(\theta)=\exp (\theta \tan (\beta \pi / 2)) .
$$

(b) Let $\alpha=\beta=0$. Then we set

$$
L^{+}(0)=\left\{e^{i t}: t \in[0,2 \pi)\right\}=\mathbb{T}, \quad L^{-}(0)=\left\{e^{i t}: t \in(-2 \pi, 0]\right\}=\mathbb{T} .
$$

(c) Let $\alpha=\beta=1$. Then we have

$$
L^{+}(1)=\left\{e^{-t}: t \geq 0\right\} \cup\{0\}=[0,1], \quad L^{-}(1)=\left\{e^{t}: t \leq 0\right\} \cup\{0\}=[0,1] .
$$

4.2. Now we introduce some sets defined by using $L^{+}(\alpha)$ and $L^{-}(\beta)$. For $\alpha, \beta \in(0,1],(\alpha, \beta) \neq(1,1)$, let

$$
\theta(\alpha, \beta)= \begin{cases}\frac{2 \pi \tan (\beta \pi / 2)}{\tan (\alpha \pi / 2)+\tan (\beta \pi / 2)}, & \alpha, \beta \in(0,1), \\ 0, & \alpha=1, \\ 2 \pi, & \beta=1 .\end{cases}
$$

(a) For $\alpha, \beta \in(0,1)$, let

$$
\begin{aligned}
& L_{0}^{+}(\alpha, \beta)=\left\{r_{\alpha}^{+}(\theta) e^{i \theta}: \theta \in[0, \theta(\alpha, \beta)]\right\}, \\
& L_{0}^{-}(\alpha, \beta)=\left\{r_{\beta}^{-}(\theta) e^{i \theta}: \theta \in[-2 \pi+\theta(\alpha, \beta), 0]\right\} .
\end{aligned}
$$

Let $L(\alpha, \beta)$ be the closed domain bounded by $L_{0}^{+}(\alpha, \beta) \cup L_{0}^{-}(\alpha, \beta)$. The sets $L_{0}^{+}(\alpha, \beta)$ and $L_{0}^{-}(\alpha, \beta)$ will be treated as oriented arcs in such a way that $L_{0}^{+}(\alpha, \beta) \cup L_{0}^{-}(\alpha, \beta)$, i.e. the boundary of $L(\alpha, \beta)$, is a positively oriented closed curve.

(b) For $\alpha, \beta \in[0,1]$ we set

$$
\begin{aligned}
& L_{0}^{+}(0)=L_{0}^{+}(0, \beta)=L_{0}^{+}(\alpha, 0)=L^{+}(0)=\mathbb{T}, \\
& L_{0}^{-}(0)=L_{0}^{-}(0, \beta)=L_{0}^{-}(\alpha, 0)=L^{-}(0)=\mathbb{T} .
\end{aligned}
$$

Then $L(0, \beta)=L(\alpha, 0)=\overline{\mathbb{D}}, \alpha, \beta \in[0,1]$.

(c) Let $L_{0}^{+}(1)=L_{0}^{+}(1,1)=L^{+}(1)=[0,1]$ and $L_{0}^{-}(1)=L_{0}^{-}(1,1)=$ $L^{-}(1)=[0,1]$. Set $L(1,1)=[0,1]$.

(d) Let $\alpha=1$ and $\beta \in(0,1)$. Now, by $(4.1), \theta(1, \beta)=0$ and let $L_{0}^{-}(1, \beta)$ be the part of $L^{-}(\beta)$ joining the points $r_{\beta}^{-}(-2 \pi)=\exp (-2 \pi \tan (\beta \pi / 2))$ and $r_{\beta}^{-}(0)=1$. We set $L_{0}^{+}(1, \beta)=[0,1]$, so $L(1, \beta)$ is the closed domain bounded by the spiral $L_{0}^{-}(1, \beta)$ and the line segment $\left[r_{\beta}^{-}(-2 \pi), 1\right]$. Its boundary is positively oriented. 
(e) Let $\beta=1$ and $\alpha \in(0,1)$. As in (d) we see by (4.1) that $\theta(\alpha, 1)$ $=2 \pi$ and let $L_{0}^{+}(\alpha, 1)$ be the part of $L^{+}(\alpha)$ joining the points $r_{\alpha}^{+}(0)=1$ and $r_{\alpha}^{+}(2 \pi)=\exp (-2 \pi \tan (\alpha \pi / 2))$. We set $L_{0}^{-}(\alpha, 1)=[0,1]$, so $L(\alpha, 1)$ is the closed domain bounded by the spiral $L_{0}^{+}(\alpha, 1)$ and the line segment $\left[r_{\alpha}^{+}(2 \pi), 1\right]$. Its boundary is positively oriented.

Note that for every $z \in L^{+}(\alpha) \backslash\{0\}, \alpha \in(0,1)$, the directed angle from $i z$ to the tangent vector to $L^{+}(\alpha) \backslash\{0\}$ at $z$ equals

$$
\arg \frac{\frac{d}{d \theta}\left(r_{\alpha}^{+}(\theta) e^{i \theta}\right)}{i r_{\alpha}^{+}(\theta) e^{i \theta}}=\arg \{i \tan (\alpha \pi / 2)+1\}=\alpha \frac{\pi}{2} .
$$

Similarly, for every $z \in L^{-}(\beta) \backslash\{0\}, \beta \in(0,1)$, the directed angle from $i z$ to the tangent vector to $L^{-}(\beta) \backslash\{0\}$ at $z$ equals

$$
\arg \frac{\frac{d}{d \theta}\left(r_{\beta}^{-}(\theta) e^{i \theta}\right)}{i r_{\beta}^{-}(\theta) e^{i \theta}}=\arg \{-i \tan (\beta \pi / 2)+1\}=-\beta \frac{\pi}{2} .
$$

Therefore we have the well known geometrical property of logarithmic spirals formulated in parts (1) and (2) of the observation below.

Observation 4.1. (1) For every $z \in L^{+}(\alpha) \backslash\{0\}, \alpha \in(0,1)$, the directed angle from $i z$ to the tangent vector to $L^{+}(\alpha) \backslash\{0\}$ at $z$ is constant and equals $\alpha \pi / 2$ (at $z=1$ we take the one-sided tangent vector to $\left.L^{+}(\alpha)\right)$.

(2) For every $z \in L^{-}(\beta) \backslash\{0\}, \beta \in(0,1)$, the directed angle from iz to the tangent vector to $L^{-}(\beta) \backslash\{0\}$ at $z$ is constant and equals $-\beta \pi / 2$ (at $z=1$ we take the one-sided tangent vector to $L^{-}(\beta)$ ).

(3) For every $z$ in $L^{+}(0)$ or in $L^{-}(0)$ the directed angle from $i z$ to the tangent vector to $L^{+}(0)$ or $L^{-}(0)$ at $z$ is constant and equals 0 .

(4) For every $z \in L^{+}(1) \backslash\{0,1\}$ (resp. $L^{-}(1) \backslash\{0,1\}$ ) the directed angle from $i z$ to the tangent vector to $L^{+}(1) \backslash\{0,1\}$ (resp. $\left.L^{-}(1) \backslash\{0,1\}\right)$ at $z$ is constant and equals $\pi / 2$ (resp. $-\pi / 2)$.

4.3. Now we define the class $\mathcal{Z}^{\supset}(\alpha, \beta)$.

Definition 4.1. A domain $\Omega \subset \mathbb{C}, \Omega \neq \mathbb{C}$, belongs to $\mathcal{Z}^{\supset}(\alpha, \beta), \alpha, \beta \in$ $[0,1]$ if $w L(\alpha, \beta) \subset \Omega$ for every $w \in \Omega$. Domains in $\mathcal{Z}^{\supset}(\alpha, \beta)$ will be called spiralshaped of order $(\alpha, \beta)$. Domains in $\mathcal{Z}^{\supset}(\alpha, \alpha), \alpha \in[0,1]$, are called spiralshaped of order $\alpha$. For short, let $\mathcal{Z}^{\supset}(\alpha)=\mathcal{Z}^{\supset}(\alpha, \alpha)$ for $\alpha \in[0,1]$.

REMARK 4.1. (1) The spiralshaped domains of order $(1,1)$ are the starlike domains, i.e. $\mathcal{Z}^{\supset}(1,1)=\mathcal{Z}^{*}$.

(2) Since $\mathcal{Z}^{\supset}(\alpha, \beta) \subset \mathcal{Z}^{*}$ for all $\alpha, \beta \in[0,1]$, every domain in $\mathcal{Z}^{\supset}(\alpha, \beta)$ is simply connected.

Since $L(0, \beta)=L(\alpha, 0)=\overline{\mathbb{D}}$ we have at once 
Observation 4.2. For any $\alpha, \beta \in[0,1]$,

$$
\mathcal{Z}^{\supset}(\alpha, 0)=\mathcal{Z}^{\supset}(0, \beta)=\left\{\mathbb{D}_{r}: r>0\right\} .
$$

The observation below has an important consequence for classes of univalent functions.

Observation 4.3. Every domain in $\mathcal{Z}^{\supset}(\alpha, \beta)$ for $\alpha, \beta \in[0,1], \quad(\alpha, \beta)$ $\neq(1,1)$, is bounded.

Proof. 1. The cases: $\alpha=0, \beta \in[0,1]$ and $\beta=0, \alpha \in[0,1]$, reduce to Observation 4.2.

2. Let $\alpha, \beta \in(0,1],(\alpha, \beta) \neq(1,1)$. Assume that $\Omega \in \mathcal{Z}^{\supset}(\alpha, \beta)$. Then $w L(\alpha, \beta) \subset \Omega$ for every $w \in \Omega$. Observe first that from strict monotonicity of the functions

$$
[0, \infty) \ni \theta \mapsto r_{\alpha}^{+}(\theta)=\exp (-\theta \tan (\alpha \pi / 2))
$$

and

$$
(-\infty, 0] \ni \theta \mapsto r_{\beta}^{-}(\theta)=\exp (\theta \tan (\beta \pi / 2))
$$

defined in Section 4.1, the set $L(\alpha, \beta)$ contains the closed disk $\overline{\mathbb{D}}_{r(\alpha, \beta)}$, where

$$
r(\alpha, \beta)=r_{\alpha}^{+}(\theta(\alpha, \beta))=r_{\beta}^{-}(2 \pi-\theta(\alpha, \beta))=\exp (-\theta(\alpha, \beta) \tan (\alpha \pi / 2)),
$$

where $\theta(\alpha, \beta)$ is given by (4.1). Thus

$$
\overline{\mathbb{D}}_{|w| r(\alpha, \beta)} \subset w L(\alpha, \beta) .
$$

Suppose now that $\Omega$ is unbounded. Then there exists a sequence $\left(w_{n}\right)$ of points of $\Omega$ such that $\lim _{n \rightarrow \infty} w_{n}=\infty$. Since $w_{n} \in \Omega$, we have $w_{n} L(\alpha, \beta)$ $\subset \Omega$ for every $n \in \mathbb{N}$. By $(4.2), \overline{\mathbb{D}}_{\left|w_{n}\right| r(\alpha, \beta)} \subset \Omega$ for every $n \in \mathbb{N}$. Since $\lim _{n \rightarrow \infty}\left|w_{n}\right|=\infty$ we deduce that $\Omega=\mathbb{C}$. Thus we have a contradiction, so $\Omega$ is bounded.

REMARK 4.2. As was noted in Remark 4.1(1), the spiralshaped domains of order $(1,1)$ are exactly the starlike domains. It is evident that starlike domains need not be bounded.

4.4. Now we prove that strong starlikeneness and spirallikeness of a given order yield the same class of plane domains. Let us start with the following observation.

Observation 4.4. $E_{k(\alpha), k(\beta)} \subset L(\alpha, \beta)$ for every $\alpha, \beta \in[0,1]$.

Proof. 1. The inclusion is evident for $\alpha=0$ or $\beta=0$, since then $L(0, \beta)=$ $L(\alpha, 0)=\overline{\mathbb{D}}, \alpha, \beta \in[0,1]$. Clearly, $E_{k(1), k(1)}=L(1,1)=[0,1]$.

2. Assume that $\alpha, \beta \in(0,1]$. Recall that the boundary of $E_{k(\alpha), k(\beta)}$ consists of two $\operatorname{arcs} \Gamma_{k(\alpha)}^{+}$and $\Gamma_{k(\beta)}^{-}$which are either circular arcs or the segment $[0,1]$. 
(a) Let first $\alpha, \beta \in(0,1)$. As in Section 2.3, $\Gamma_{k(\alpha)}^{+} \backslash\{0\}$ and $\Gamma_{k(\beta)}^{-} \backslash\{0\}$ with $k=k(\alpha)=2 \cos (\alpha \pi / 2)$ and $l=k(\beta)=2 \cos (\beta \pi / 2)$ have the polar equations (2.3) and (2.4), respectively. Moreover,

$$
\begin{aligned}
\left.\varrho_{k(\alpha)}^{+}((1-\alpha) \pi / 2)\right) & =\lim _{\theta \rightarrow[(1-\alpha) \pi / 2]^{-}} \varrho_{k(\alpha)}^{+}(\theta)=0, \\
\left.\varrho_{k(\beta)}^{-}(-(1-\beta) \pi / 2)\right) & =\lim _{\theta \rightarrow[-(1-\beta) \pi / 2]^{+}} \varrho_{k(\beta)}^{-}(\theta)=0 .
\end{aligned}
$$

We will show that

$$
a(\theta):=r_{\alpha}^{+}(\theta)-\varrho_{k(\alpha)}^{+}(\theta) \geq 0
$$

for $\theta \in[0,(1-\alpha) \pi / 2]$, where $r_{\alpha}^{+}(\theta)$ is given in 4.1. Since

$$
\begin{aligned}
a^{\prime}(\theta) & =\frac{1}{\cos (\alpha \pi / 2)}(\sin (\theta+\alpha \pi / 2)-\exp (-\theta \tan (\alpha \pi / 2)) \sin (\alpha \pi / 2)) \\
& \geq \frac{1}{\cos (\alpha \pi / 2)}(\sin (\theta+\alpha \pi / 2)-\sin (\alpha \pi / 2))>0
\end{aligned}
$$

for $\theta \in(0,(1-\alpha) \pi / 2)$, the function $a$ is strictly increasing in $(0,(1-\alpha) \pi / 2)$. Moreover $a(0)=r_{\alpha}^{+}(0)-\varrho_{k(\alpha)}^{+}(0)=0$ and $a((1-\alpha) \pi / 2)>0$. This yields (4.3).

In a similar way we show $r_{\beta}^{-}(\theta)-\varrho_{k(\beta)}^{-}(\theta) \geq 0$ for $\theta \in[-(1-\beta) \pi / 2,0]$. Consequently, $E_{k(\alpha), k(\beta)}$ lies in the intersection of $L(\alpha, \beta)$ and the closed convex angle with vertex at zero and arms $l[0,(1-\alpha) \pi / 2]$ and $l[0,-(1-\beta) \pi / 2]$. In particular, $E_{k(\alpha), k(\beta)} \subset L(\alpha, \beta)$.

(b) Let $\beta=1$ and $\alpha \in(0,1)$. Then $E_{k(\alpha), k(1)}$ is the closed domain bounded by the circular arc $\Gamma_{k(\alpha)}^{+}$and the line segment [0,1], and $L(\alpha, 1)$ is the closed domain bounded by the spiral $L_{0}^{+}(\alpha, 1)$ and the segment $\left[r_{\alpha}^{+}(2 \pi), 1\right]$. As in part 2(a) we show (4.3). Thus $E_{k(\alpha), k(1)}$ lies in the intersection of $L(\alpha, 1)$ and the closed convex angle with vertex at zero and arms $l[0,(1-\alpha) \pi / 2]$ and $l[0,0]$. In particular, $E_{k(\alpha), k(1)} \subset L(\alpha, 1)$.

Similarly we prove the case $\alpha=1$ and $\beta \in(0,1)$.

From Observation 4.4 we have

Corollary 4.1. $\mathcal{Z}^{\supset}(\alpha, \beta) \subset \mathcal{Z}^{*}(\alpha, \beta)$ for all $\alpha, \beta \in[0,1]$.

Now we prove the reverse inclusion.

Lemma 4.1. $\mathcal{Z}^{*}(\alpha, \beta) \subset \mathcal{Z}^{\supset}(\alpha, \beta)$ for all $\alpha, \beta \in[0,1]$.

Proof. 1 . The case $\alpha=\beta=1$ is evident since $\mathcal{Z}^{*}(1,1)=\mathcal{Z}^{\supset}(1,1)=\mathcal{Z}^{*}$.

The case $\alpha=0$ and $\beta \in[0,1](\beta=0$ and $\alpha \in[0,1])$ follows from Lemma 2.2 and Observation 4.2. Then

$$
\mathcal{Z}^{*}(0, \beta)=\mathcal{Z}^{\supset}(0, \beta)=\mathcal{Z}^{*}(\alpha, 0)=\mathcal{Z}^{\supset}(\alpha, 0)=\left\{\mathbb{D}_{r}: r>0\right\} .
$$


2. Assume that $\alpha, \beta \in(0,1],(\alpha, \beta) \neq(1,1)$. Let $\Omega \in \mathcal{Z}^{*}(\alpha, \beta)$. Then $w E_{k(\alpha), k(\beta)} \subset \Omega$ for every $w \in \Omega$. We prove that $w L(\alpha, \beta) \subset \Omega$.

Fix $w \in \Omega$. Without loss of generality we can assume that $w=r \in \mathbb{R}_{+}$.

(a) Assume first that $\beta \in(0,1)$. Then $r \Gamma_{k(\beta)}^{-} \subset \Omega$. We now prove that $r L^{-}(\beta) \subset \Omega$, i.e. for every $t \leq 0$,

$$
r e^{i t} \exp (t \tan (\beta \pi / 2)) \in \Omega .
$$

To this end, fix $t<0$ and $n \in \mathbb{N}$ such that $t / n>-(1-\beta) \pi / 2$. Since $r E_{k(\alpha), k(\beta)} \subset \Omega$, by a simple computation we have

$$
w_{1}=e^{i t / n} r(\cos (t / n)-\sin (t / n) \tan (\beta \pi / 2)) \in r \Gamma_{k(\beta)}^{-} .
$$

Consequently, $w_{1} \Gamma_{k(\beta)}^{-} \subset \Omega$ since $w_{1} \in \Omega$. Hence

$$
\begin{aligned}
w_{2} & =e^{2 i t / n}\left|w_{1}\right|(\cos (t / n)-\sin (t / n) \tan (\beta \pi / 2)) \\
& =e^{2 i t / n} r(\cos (t / n)-\sin (t / n) \tan (\beta \pi / 2))^{2} \in w_{1} \Gamma_{k(\beta)}^{-} .
\end{aligned}
$$

Clearly, $w_{2} \in \Omega$. In this way we can find $n+1$ points $w_{0}, w_{1}, w_{2}, \ldots, w_{n}$ of $\Omega$ such that $w_{0}=r$ and

$$
\begin{aligned}
w_{k} & =e^{i k t / n}\left|w_{k-1}\right|(\cos (t / n)-\sin (t / n) \tan (\beta \pi / 2)) \\
& =e^{i k t / n} r(\cos (t / n)-\sin (t / n) \tan (\beta \pi / 2))^{k} \in w_{k-1} \Gamma_{k(\beta)}^{-}
\end{aligned}
$$

for $k=1, \ldots, n$. Since, in particular,

$$
w_{n}=e^{i t} r(\cos (t / n)-\sin (t / n) \tan (\beta \pi / 2))^{n}
$$

we have

$$
\begin{aligned}
\lim _{n \rightarrow \infty} w_{n}= & e^{i t} r \lim _{n \rightarrow \infty}(\cos (t / n)-\sin (t / n) \tan (\beta \pi / 2))^{n} \\
= & e^{i t} r \lim _{n \rightarrow \infty} \exp (n \log (\cos (t / n)-\sin (t / n) \tan (\beta \pi / 2))) \\
= & e^{i t} r \lim _{n \rightarrow \infty} \exp \left(-n\left(2 \sin ^{2}(t / 2 n)-\sin (t / n) \tan (\beta \pi / 2)\right)\right. \\
& \left.\times \frac{\left.\log \left(1-2 \sin ^{2}(t / 2 n)-\sin (t / n) \tan (\beta \pi / 2)\right)\right)}{-2 \sin ^{2}(t / 2 n)-\sin (t / n) \tan (\beta \pi / 2)}\right) \\
= & r e^{i t} \exp (t \tan (\beta \pi / 2)) .
\end{aligned}
$$

Thus we showed that $r L^{-}(\beta) \subset \bar{\Omega}$. Since also $r_{1} L^{-}(\beta) \subset \bar{\Omega}$ for every positive $r_{1}$ in $\Omega$ such that $r_{1}>r$, we deduce at once that $r L^{-}(\beta) \subset \Omega$.

By the same argument we show that $r L^{+}(\alpha) \subset \Omega$ when $\alpha \in(0,1)$. Consequently, $r L(\alpha, \beta) \subset \Omega$ for $\alpha, \beta \in(0,1)$. 
(b) Let $\alpha=1$ and $\beta \in(0,1)$. As in part 2(a) we show that $r L^{-}(\beta) \subset \Omega$. Since moreover $r L^{+}(1)=[0, r] \subset \Omega$, we see that $L(1, \beta) \subset \Omega$.

The case of $\beta=1$ and $\alpha \in(0,1)$ is similar.

Thus the proof of the lemma is finished.

Corollary 4.1 and Lemma 4.1 yield

Theorem 4.1. $\mathcal{Z}^{*}(\alpha, \beta)=\mathcal{Z}^{\supset}(\alpha, \beta)$ for all $\alpha, \beta \in[0,1]$.

From the above and from Observation 4.3 we have

Corollary 4.2. Every domain in $\mathcal{Z}^{*}(\alpha, \beta)$ for $\alpha, \beta \in[0,1],(\alpha, \beta)$ $\neq(1,1)$, is bounded.

\section{Spirallike functions of order $(\alpha, \beta)$}

5.1. For $\alpha, \beta \in[0,1]$ let $\mathcal{S}^{\supset}(\alpha, \beta) \subset \mathcal{S}$ be the class of functions $f$ such that $f(\mathbb{D}) \in \mathcal{Z}^{\supset}(\alpha, \beta)$. Functions in $\mathcal{S}^{\supset}(\alpha, \beta)$ for $\alpha, \beta \in[0,1]$ are called spirallike of order $(\alpha, \beta)$. Let $\mathcal{S}^{\supset}(\alpha, \beta ; \xi)=\mathcal{S}^{\supset}(\alpha, \beta) \cap \mathcal{S}(\xi)$ and $\mathcal{S}^{\supset}(\alpha)=$ $\mathcal{S}^{\supset}(\alpha, \alpha)$. Functions in $\mathcal{S}^{\supset}(\alpha)$ are called spirallike of order $\alpha$.

By Lemma 2.2 and Observation 4.2, for $\alpha, \beta \in[0,1]$, we have

$$
\mathcal{S}^{\supset}(0, \beta ; \xi)=\mathcal{S}^{*}(0, \beta ; \xi)=\mathcal{S}^{\supset}(\alpha, 0 ; \xi)=\mathcal{S}^{*}(\alpha, 0 ; \xi)=\left\{a \varphi_{\xi}: a \in \mathbb{C} \backslash\{0\}\right\} .
$$

5.2. The next two theorems are fundamental for the concepts of strong starlikeness and spirallikeness. The first one is an immediate consequence of Theorem 4.1, and the second, of Observation 4.3 and Corollary 4.2. These results are known for $\alpha=\beta \in(0,1)$ but their proofs based on some properties of strongly starlike functions of order $\alpha$ which follow from their analytic formula are nonelementary (see e.g. [Su]). Theorem 5.3 for the class $\mathcal{S}^{*}(\alpha), \alpha \in(0,1)$, was proved in [BK].

Theorem 5.1. If $\alpha, \beta \in(0,1]$ and $\xi \in \mathbb{D}$, then

$$
\mathcal{S}^{\supset}(\alpha, \beta ; \xi)=\mathcal{S}^{*}(\alpha, \beta ; \xi) .
$$

Theorem 5.2. Every function in $\mathcal{S}^{*}(\alpha, \beta)$ and in $\mathcal{S}^{\supset}(\alpha, \beta)$ for $\alpha, \beta \in$ $(0,1]$ with $(\alpha, \beta) \neq(1,1)$, is bounded.

Summarizing we have

Theorem 5.3. Let $\alpha, \beta \in(0,1], \xi \in \mathbb{D}$ and $f \in \mathcal{A}$ with $f^{\prime}(\xi) \neq 0$. Then the following conditions are equivalent:

(1) $f$ satisfies (3.1);

(2) $f \in \mathcal{S}^{*}(\alpha, \beta ; \xi)$;

(3) $f \in \mathcal{S}^{\supset}(\alpha, \beta ; \xi)$.

TheOREm 5.4. Let $\alpha, \beta \in(0,1]$ and $\Omega \subset \mathbb{C}, \Omega \neq \mathbb{C}$, be a simply connected domain. Then the following conditions are equivalent: 
(1) For each $\xi \in \mathbb{D}$ there exists $f \in \mathcal{A}(\xi)$ with $f^{\prime}(\xi) \neq 0$ such that $\Omega=f(\mathbb{D})$ and $f$ satisfies $(3.1)$. In particular, $\Omega=f(\mathbb{D})$ for some $f \in \mathcal{A}(0)$, with $f^{\prime}(0) \neq 0$, satisfying (3.22);

(2) $\Omega \in \mathcal{Z}^{*}(\alpha, \beta)$;

(3) $\Omega \in \mathcal{Z}^{\supset}(\alpha, \beta)$.

\section{Meromorphic strongly starlike and spirallike functions of order $(\alpha, \beta)$}

6.1. For $\alpha, \beta \in[0,1]$ let

$$
E_{\alpha, \beta}(\infty)=\left\{w \in \mathbb{C}:-(1-\alpha) \frac{\pi}{2} \leq \arg (w-1) \leq(1-\beta) \frac{\pi}{2}\right\} \cup\{1, \infty\} .
$$

Definition 6.1. A simply connected domain $\Omega \subset \overline{\mathbb{C}}, \Omega \neq \overline{\mathbb{C}}$, belongs to the class $\mathcal{Z}^{*}(\alpha, \beta ; \infty), \alpha, \beta \in[0,1]$, and will be called strongly starlike of order $(\alpha, \beta)$ with respect to infinity if $w E_{\alpha, \beta}(\infty) \subset \Omega$ for every $w \in \Omega$.

Let $\mathcal{Z}^{*}(\alpha ; \infty)=\mathcal{Z}^{*}(\alpha, \alpha ; \infty), \alpha \in[0,1]$, denote the class of strongly starlike domains of order $\alpha$ with respect to infinity.

Let $\mathcal{Z}^{*}(\infty)=\mathcal{Z}^{*}(1 ; \infty)$ denote the class of starlike domains with respect to infinity (as an interior point).

6.2. For $\alpha, \beta \in[0,1]$ we denote by $L^{+}(\alpha ; \infty)$ and $L^{-}(\beta ; \infty)$ the closures in $\overline{\mathbb{C}}$ of the sets $\left\{\exp \left(e^{-i(1-\alpha) \pi / 2} t\right): t \geq 0\right\}$ and $\left\{\exp \left(-e^{i(1-\beta) \pi / 2} t\right): t \leq 0\right\}$, respectively.

(a) Let $\alpha, \beta \in(0,1)$. Then $L^{+}(\alpha ; \infty)$ and $L^{-}(\beta ; \infty)$ are two logarithmic spirals closed in $\overline{\mathbb{C}}$ joining 1 and $\infty$. Let us write $L^{+}(\alpha ; \infty) \backslash\{\infty\}$ and $L^{-}(\beta ; \infty) \backslash\{\infty\}$ in polar coordinates:

$$
\begin{array}{lll}
L^{+}(\alpha ; \infty) \backslash\{\infty\}: & z=r_{\alpha}^{+}(\theta ; \infty) e^{-i \theta}, & \theta \in[0, \infty), \\
L^{-}(\beta ; \infty) \backslash\{\infty\}: & z=r_{\beta}^{-}(\theta ; \infty) e^{-i \theta}, & \theta \in(-\infty, 0],
\end{array}
$$

where

$$
r_{\alpha}^{+}(\theta ; \infty)=\exp (\theta \tan (\alpha \pi / 2)), \quad r_{\beta}^{-}(\theta ; \infty)=\exp (-\theta \tan (\beta \pi / 2)) .
$$

We see that $L^{+}(\alpha ; \infty)$ is oriented from 1 to $\infty$, and $L^{-}(\beta ; \infty)$ from $\infty$ to 1 .

(b) Let $\alpha=\beta=0$. Then we set

$$
\begin{aligned}
& L^{+}(0 ; \infty)=\left\{e^{-i t}: t \in[0,2 \pi)\right\}=\mathbb{T}, \\
& L^{-}(0 ; \infty)=\left\{e^{-i t}: t \in(-2 \pi, 0]\right\}=\mathbb{T} .
\end{aligned}
$$

(c) Let $\alpha=\beta=1$. Then we have

$$
L^{+}(1 ; \infty)=\left\{e^{t}: t \geq 0\right\} \cup\{\infty\}, \quad L^{-}(1 ; \infty)=\left\{e^{-t}: t \leq 0\right\} \cup\{\infty\} .
$$


6.3. Now we introduce some sets defined via $L^{+}(\alpha ; \infty)$ and $L^{-}(\beta ; \infty)$.

(a) For $\alpha, \beta \in(0,1)$, let

$$
\begin{aligned}
& L_{0}^{+}(\alpha, \beta ; \infty)=\left\{r_{\alpha}^{+}(\theta ; \infty) e^{-i \theta}: \theta \in[0, \theta(\alpha, \beta)]\right\}, \\
& L_{0}^{-}(\alpha, \beta ; \infty)=\left\{r_{\beta}^{-}(\theta ; \infty) e^{-i \theta}: \theta \in[-2 \pi+\theta(\alpha, \beta), 0]\right\},
\end{aligned}
$$

where $\theta(\alpha, \beta)$ is given by $(4.1)$.

Let $L(\alpha, \beta ; \infty)$ be the complement in $\overline{\mathbb{C}}$ of the domain bounded by $L_{0}^{+}(\alpha, \beta ; \infty) \cup L_{0}^{-}(\alpha, \beta ; \infty)$.

(b) For $\alpha, \beta \in[0,1]$ we set

$$
\begin{aligned}
& L_{0}^{+}(0 ; \infty)=L_{0}^{+}(0, \beta ; \infty)=L_{0}^{+}(\alpha, 0 ; \infty)=L^{+}(0 ; \infty)=\mathbb{T}, \\
& L_{0}^{-}(0 ; \infty)=L_{0}^{-}(0, \beta ; \infty)=L_{0}^{-}(\alpha, 0 ; \infty)=L^{-}(0 ; \infty)=\mathbb{T} .
\end{aligned}
$$

Then $L(0, \beta ; \infty)=L(\alpha, 0 ; \infty)=\overline{\mathbb{D}}^{*}, \alpha, \beta \in[0,1]$.

(c) Let $L_{0}^{+}(1 ; \infty)=L_{0}^{+}(1,1 ; \infty)=L^{+}(1 ; \infty)=[1, \infty) \cup\{\infty\}$ and let $L_{0}^{-}(1 ; \infty)=L_{0}^{-}(1,1 ; \infty)=L^{-}(1 ; \infty)=[1, \infty) \cup\{\infty\}$. Set $L(1,1 ; \infty)=$ $[1, \infty) \cup\{\infty\}$.

(d) Let $\alpha=1$ and $\beta \in(0,1)$. Now, by (4.1) we see that $\theta(1, \beta)=0$ and let $L_{0}^{-}(1, \beta ; \infty)$ be the part of $L^{-}(\beta ; \infty)$ joining the points $r_{\beta}^{-}(-2 \pi ; \infty)=$ $\exp (2 \pi \tan (\beta \pi / 2))$ and $r_{\beta}^{-}(0 ; \infty)=1$. We set $L_{0}^{+}(1, \beta ; \infty)=[1, \infty) \cup\{\infty\}$, so $L(1, \beta ; \infty)$ is the complement in $\overline{\mathbb{C}}$ of the domain bounded by $L_{0}^{+}(1, \beta ; \infty)$ and the line segment $\left[1, r_{\beta}^{-}(-2 \pi ; \infty)\right]$.

(e) Let $\beta=1$ and $\alpha \in(0,1)$. By $(4.1)$ we see that $\theta(\alpha, 1)=2 \pi$ and let $L_{0}^{+}(\alpha, 1 ; \infty)$ be the part of $L^{+}(\alpha ; \infty)$ joining the points $r_{\alpha}^{-}(0 ; \infty)=1$ and $r_{\alpha}^{+}(2 \pi ; \infty)=\exp (2 \pi \tan (\beta \pi / 2))$. We set $L_{0}^{-}(\alpha, 1 ; \infty)=[1, \infty) \cup\{\infty\}$, so $L(\alpha, 1 ; \infty)$ is the complement in $\overline{\mathbb{C}}$ of the domain bounded by the line segment $\left[1, r_{\beta}^{-}(-2 \pi ; \infty)\right]$ and $L_{0}^{-}(1 ; \infty)$.

Since $L(\alpha, \beta ; \infty) \in \mathcal{E}_{\infty}$ we can formulate the following definition.

Definition 6.2. A simply connected domain $\Omega \subset \overline{\mathbb{C}}, \Omega \neq \overline{\mathbb{C}}$, belongs to the class $\mathcal{Z}^{\supset}(\alpha, \beta ; \infty), \alpha, \beta \in[0,1]$, and will be called spiralshaped of order $(\alpha, \beta)$ with respect to infinity if $w L(\alpha, \beta ; \infty) \subset \Omega$ for every $w \in \Omega$.

Let $\mathcal{Z}^{\supset}(\alpha ; \infty)=\mathcal{Z}^{\supset}(\alpha, \alpha ; \infty), \alpha \in[0,1]$, be the class of spiralshaped domains of order $\alpha$ with respect to infinity.

Clearly, $\mathcal{Z}^{\supset}(1 ; \infty)=\mathcal{Z}^{*}(\infty)$.

Since $L(0, \beta ; \infty)=L(\alpha, 0 ; \infty)=\overline{\mathbb{D}}^{*}$ we have at once

Observation 6.1. (1) For all $\alpha, \beta \in[0,1]$,

$$
\mathcal{Z}^{\supset}(\alpha, 0 ; \infty)=\mathcal{Z}^{\supset}(0, \beta ; \infty)=\left\{\mathbb{D}_{r}^{*}: r>0\right\} .
$$


ObSERVATion 6.2. Let $\alpha, \beta \in[0,1]$. Then

(1) $0 \notin \Omega$ for every $\Omega \in \mathcal{Z}^{*}(\alpha, \beta ; \infty)$;

(2) $0 \notin \Omega$ for every $\Omega \in \mathcal{Z}^{\supset}(\alpha, \beta ; \infty)$.

Proof. (1) Indeed, assume that $0 \in \Omega$ for some $\Omega \in \mathcal{Z}^{*}(\alpha, \beta ; \infty)$. Since $\Omega$ is a proper subdomain of $\overline{\mathbb{C}}$, there exists a finite point $w_{0} \in \partial \Omega$ such that $\left[0, w_{0}\right) \subset \Omega$. Hence the sector $w E_{\alpha, \beta}(\infty)$ does not lie in $\Omega$ for any point $w$ of the segment $\left[0, w_{0}\right)$.

(2) Similar.

The facts below are clear.

ObSERVATion 6.3. Let $\alpha, \beta \in[0,1]$. Then

(1) $E_{\alpha, \beta}(\infty)=\left(E_{k(\alpha), k(\beta)}\right)^{-1}$;

(2) $L(\alpha, \beta ; \infty)=(L(\alpha, \beta))^{-1}$;

(3) $\Omega \in \mathcal{Z}^{*}(\alpha, \beta ; \infty)$ if and only if $\Omega^{-1} \in \mathcal{Z}^{*}(\alpha, \beta)$;

(4) $\Omega \in \mathcal{Z}^{\supset}(\alpha, \beta ; \infty)$ if and only if $\Omega^{-1} \in \mathcal{Z}^{\supset}(\alpha, \beta)$.

Let $\Omega \in \mathcal{Z}^{*}(\alpha, 0 ; \infty)$ for some $\alpha \in[0,1]$. Then $\Omega^{-1} \in \mathcal{Z}^{*}(\alpha, 0)$ by Observation 6.3(3). By Lemma 2.2, $\Omega^{-1}=\mathbb{D}_{r}$ for some positive $r$. Thus $\Omega=\mathbb{D}_{1 / r}^{*}$. Hence we have

Observation 6.4. For all $\alpha, \beta \in[0,1]$,

$$
\mathcal{Z}^{*}(\alpha, 0 ; \infty)=\mathcal{Z}^{*}(0, \beta ; \infty)=\left\{\mathbb{D}_{r}^{*}: r>0\right\} .
$$

6.4. For $\alpha, \beta \in[0,1]$ let $\Sigma^{*}(\alpha, \beta) \subset \Sigma$ be the class of all functions $g$ such that $g\left(\mathbb{D}^{*}\right) \in \mathcal{Z}^{*}(\alpha, \beta ; \infty)$, and let $\Sigma^{\partial}(\alpha, \beta) \subset \Sigma$ be the class of all functions $g$ such that $g\left(\mathbb{D}^{*}\right) \in \mathcal{Z}^{\supset}(\alpha, \beta ; \infty)$. Such functions will be called meromorphic strongly starlike and meromorphic spirallike of order $(\alpha, \beta)$, respectively. For $\alpha \in[0,1]$, let $\Sigma^{*}(\alpha)=\Sigma^{*}(\alpha, \alpha)$ be the class of functions meromorphic strongly starlike of order $\alpha$, and let $\Sigma^{\mathrm{D}}(\alpha)=\Sigma^{\mathrm{D}}(\alpha, \alpha)$ be the class of functions meromorphic spirallike of order $\alpha$. Clearly, $\Sigma^{*}(1)=\Sigma^{\circlearrowright}(1)=\Sigma^{*}$ is the class of meromorphic starlike functions. In view of Observation 6.2, $\Sigma^{*}(\alpha, \beta)$ and $\Sigma^{\circlearrowright}(\alpha, \beta)$ are subsets of $\Sigma^{\prime}$.

To find an analytic characterization of functions in $\Sigma^{*}(\alpha, \beta)$ or $\Sigma^{\supset}(\alpha, \beta)$ we can use results of the previous sections.

For $\alpha, \beta \in[0,1]$ from Observations 6.1 and 6.4 we have

$$
\begin{aligned}
\Sigma^{*}(\alpha, 0) & =\Sigma^{*}(0, \beta)=\Sigma^{\supset}(\alpha, 0)=\Sigma^{\supset}(0, \beta) \\
& =\left\{b \zeta: \zeta \in \mathbb{D}^{*}, b \in \mathbb{C} \backslash\{0\}\right\} .
\end{aligned}
$$

Therefore assume that $\alpha, \beta \in(0,1]$. Let $\Omega \in \mathcal{Z}^{*}(\alpha, \beta ; \infty)$ (resp. $\left.\mathcal{Z}^{\supset}(\alpha, \beta ; \infty)\right)$ and $g \in \Sigma^{*}(\alpha, \beta)$ (resp. $\left.\Sigma^{\supset}(\alpha, \beta ; \infty)\right)$ be such that $g\left(\mathbb{D}^{*}\right)=\Omega$. By Observation 6.3(3) we see that $\Omega^{-1} \in \mathcal{Z}^{*}(\alpha, \beta)$ (resp. $\mathcal{Z}^{\supset}(\alpha, \beta)$ ). Moreover $f=\hbar \circ g \circ \hbar$ belongs to $\mathcal{S}^{*}(\alpha, \beta ; 0)\left(\operatorname{resp} . \mathcal{S}^{\supset}(\alpha, \beta ; 0)\right)$, and $f(\mathbb{D})=\Omega^{-1}$. For this reason using Corollary 3.2 and Theorems $5.3-5.4$ for $\xi=0$ we have 
Theorem 6.1. Let $\alpha, \beta \in(0,1]$ and $g$ be of the form

$$
g(\zeta)=b \zeta+\sum_{n=0}^{\infty} \frac{b_{n}}{\zeta^{n}}, \quad \zeta \in \mathbb{D}^{*}, b \in \mathbb{C} \backslash\{0\} .
$$

Then the following conditions are equivalent:

(1) $g$ satisfies the condition

$$
-\beta \frac{\pi}{2}<\arg \left\{\frac{\zeta g^{\prime}(\zeta)}{g(\zeta)}\right\}<\alpha \frac{\pi}{2}, \quad \zeta \in \mathbb{D}^{*} ;
$$

(2) $g \in \Sigma^{*}(\alpha, \beta)$;

(3) $g \in \Sigma^{\mathrm{D}}(\alpha, \beta)$.

TheOREM 6.2. Let $\alpha, \beta \in(0,1]$ and $\Omega \subset \overline{\mathbb{C}}, \Omega \neq \overline{\mathbb{C}}$, be a simply connected domain. Then the following conditions are equivalent:

(1) There exists a meromorphic function $g$ of the form (6.1) such that $\Omega=g\left(\mathbb{D}^{*}\right)$ and $g$ satisfies $(6.2)$;

(2) $\Omega \in \mathcal{Z}^{*}(\alpha, \beta ; \infty)$;

(3) $\Omega \in \mathcal{Z}^{\supset}(\alpha, \beta ; \infty)$.

6.5. Now we summarize the results of this section.

Theorem 6.3. Let $\alpha, \beta \in(0,1],(\alpha, \beta) \neq(1,1)$, and $\Omega \subset \mathbb{C}, \Omega \neq \mathbb{C}$, be a simply connected domain. Then the following conditions are equivalent:

(1) For each $\xi \in \mathbb{D}$ there exists $f \in \mathcal{A}(\xi)$ with $f^{\prime}(\xi) \neq 0$ such that $\Omega=f(\mathbb{D})$ and $f$ satisfies $(3.1)$;

(2) There exists a meromorphic function $g$ of the form (6.1) such that $\operatorname{Ext} \Omega=g\left(\mathbb{D}^{*}\right)$ and $g$ satisfies (6.2);

(3) $\Omega \in \mathcal{Z}^{*}(\alpha, \beta)$;

(4) $\Omega \in \mathcal{Z}^{\supset}(\alpha, \beta)$;

(5) Ext $\Omega \in \mathcal{Z}^{*}(\alpha, \beta ; \infty)$;

(6) $\operatorname{Ext} \Omega \in \mathcal{Z}^{\supset}(\alpha, \beta ; \infty)$.

Proof. (a) This theorem is known for $\beta=\alpha \in(0,1)$ (see e.g. [Su]) and summarizes the knowledge about the class of strongly starlike functions of order $\alpha$.

The method of proof presented here for the class of strongly starlike functions of order $(\alpha, \beta)$ is elementary and different from that in $[\mathrm{Su}]$. Theorem 5.4 yields the equivalences $(1) \Leftrightarrow(3) \Leftrightarrow(4)$, and Theorem 6.2 yields the equivalences $(2) \Leftrightarrow(5) \Leftrightarrow(6)$. Now we prove that $(4) \Leftrightarrow(6)$ using only geometrical arguments.

(b) Let $\Omega \in \mathcal{Z}^{\supset}(\alpha, \beta)$. We prove first that $w L(\alpha, \beta) \subset \bar{\Omega}$ for every $w \in \bar{\Omega}$. This is evident for $w \in \Omega$ since then $w L(\alpha, \beta) \subset \Omega$. Therefore let $w \in \partial \Omega$. Assume that $w_{0} \in w L(\alpha, \beta)$, i.e. $w_{0}=w u_{0}$ for some $u_{0} \in L(\alpha, \beta)$. Since $w \in \partial \Omega$, there exists a sequence $\left(w_{n}\right)$ of points in $\Omega$ convergent to $w$. The 
inclusion $w_{n} L(\alpha, \beta) \subset \Omega$ shows that $w_{n} u_{0} \in \Omega$ for every $n \in \mathbb{N}$. Since $\lim _{n \rightarrow \infty}\left(w_{n} u_{0}\right)=w u_{0}=w_{0}$, we see that our claim holds. In particular, $w L^{+}(\alpha) \subset \bar{\Omega}$ and $w L^{-}(\beta) \subset \bar{\Omega}$ for every $w \in \bar{\Omega}$.

(c) The domain $\Omega$ is bounded as was shown in Observation 4.3. Therefore $\operatorname{Ext} \Omega \neq \emptyset$ and $\infty \in \operatorname{Ext} \Omega$. Now we prove that $w L^{+}(\alpha ; \infty) \subset \operatorname{Ext} \Omega$ and $w L^{-}(\beta ; \infty) \subset \operatorname{Ext} \Omega$ for every $w \in \operatorname{Ext} \Omega$. Indeed, let $w \in \operatorname{Ext} \Omega$. Suppose, on the contrary, that there exists a point $w_{0} \in w L^{+}(\alpha ; \infty)$ such that $w_{0} \notin$ Ext $\Omega$. Since $w_{0} \in w L^{+}(\alpha ; \infty)$, there exists $t_{0}>0$ such that

$$
w_{0}=w \exp \left(e^{-i(1-\alpha) \pi / 2} t_{0}\right) .
$$

Hence

$$
w=w_{0} \exp \left(-e^{-i(1-\alpha) \pi / 2} t_{0}\right),
$$

which means that $w \in w_{0} L^{+}(\alpha)$.

Since $w_{0} \notin \operatorname{Ext} \Omega$, we see that $w_{0} \in \bar{\Omega}$ and by part (2) of this proof we deduce that $w_{0} L^{+}(\alpha) \subset \bar{\Omega}$. But $w \in w_{0} L^{+}(\alpha)$ so $w \in \bar{\Omega}$, which yields a contradiction.

Similarly we prove that $w L^{-}(\beta ; \infty) \subset \operatorname{Ext} \Omega$.

(d) Let $w \in \operatorname{Ext} \Omega$. Recall from Section 6.3 the spirals $w L_{0}^{+}(\alpha, \beta ; \infty) \subset$ $w L^{+}(\alpha ; \infty)$ and $w L_{0}^{-}(\alpha, \beta ; \infty) \subset w L^{-}(\beta ; \infty)$. Thus by part (c) of this proof, $w L_{0}^{+}(\alpha, \beta ; \infty) \subset$ Ext $\Omega$ and $w L_{0}^{-}(\alpha, \beta ; \infty) \subset \operatorname{Ext} \Omega$. Consequently, $\Omega$ lies in the interior of the bounded set with $w L_{0}^{+}(\alpha, \beta ; \infty) \cup w L_{0}^{-}(\alpha, \beta ; \infty)$ as its boundary. Since $w L_{0}^{+}(\alpha, \beta ; \infty) \cup w L_{0}^{-}(\alpha, \beta ; \infty)$ is the boundary of $w L(\alpha, \beta ; \infty)$, we deduce that $w L(\alpha, \beta ; \infty) \subset \operatorname{Ext} \Omega$. This proves that Ext $\Omega \in \mathcal{Z}^{\supset}(\alpha, \beta ; \infty)$.

(e) Similarly, we show that if Ext $\Omega \in \mathcal{Z}^{\supset}(\alpha, \beta ; \infty)$, then $\Omega \in \mathcal{Z}^{\supset}(\alpha, \beta)$. This ends the proof of the theorem.

\section{Remarks}

7.1. Theorem 3.2 characterizes analytically the class $\mathcal{S}^{*}(\alpha, \beta ; \xi)$ of strongly starlike functions of order $(\alpha, \beta)$. We have shown that the same analytic condition (3.1) describes the class $\mathcal{S}^{\curvearrowright}(\alpha, \beta ; \xi)$ of spirallike functions of order $(\alpha, \beta)$. For $\xi=0,(3.1)$ reduces to (3.22) and defines the class of functions introduced by Bucka and Ciozda $[\mathrm{BC}]$. Now this class comes equipped with a geometric interpretation.

For $\beta=\alpha \in(0,1]$ and $\xi=0$ Theorem 3.2 was proved by Brannan and Kirwan $[\mathrm{BK}]$ whereas Theorem 5.3 can be found in $[\mathrm{SW}]$ and $[\mathrm{Su}]$.

7.2. Wald $[\mathrm{W}]$ considered the class $\mathcal{S T}(\xi) \subset \mathcal{S}, \xi \in \mathbb{D}$, of functions $f$ standardly normalized by $f(0)=f^{\prime}(0)-1=0$ and such that $f(\mathbb{D})$ is a starlike domain with respect to $f(\xi)$. For details see [G, Vol. I, pp. 155-164]. 
He proved that

$$
\operatorname{Re}\left\{\frac{(1-\bar{\xi} z)(z-\xi) f^{\prime}(z)}{f(z)-f(\xi)}\right\}>0, \quad z \in \mathbb{D}
$$

is a necessary and sufficient condition for $f$ to be in $\mathcal{S T}(\xi)$. Clearly, (3.1) reduces to $(7.1)(f(\xi)=0)$ for $\alpha=\beta=1$. In fact, Wald transformed the condition

$$
\operatorname{Re}\left\{\frac{z f^{\prime}(z)}{f(z)-f(\xi)}\right\}>0, \quad z \in \mathbb{D},
$$

into the form (7.1) (see [G, Vol. I, p. 156]). The inequality (7.2) means that the domains $f\left(\mathbb{D}_{r}\right)$ are starlike with respect to $f(\xi)$ for all $r$ such that $|\xi|<r<1$. Note that at $z=\xi$ the expression on the left-hand side of (7.2) has a pole. For more about the condition (7.2) see [G, Vol. I, p. 156].

7.3. Classes of meromorphic univalent functions have been intensively studied by various authors. The condition (6.2) for $\alpha=\beta=1$ describes analytically the class $\Sigma^{*}$ of meromorphic starlike functions (see e.g. [G, Vol. II, pp. 230-232]). The case $\alpha=\beta \in(0,1]$ in (6.2) yields the class $\Sigma^{*}(\alpha)$ of meromorphic starlike functions of order $\alpha$ (see e.g. [G, Vol. II, pp. 233-235]).

7.4. For $\beta=\alpha \in(0,1)$ and $\xi=0$ Theorem 6.3 appeared in [Su]. In this case, the equivalence of (1), (5) and (6) was proved by J. Stankiewicz in [S1]; the equivalence of (1) and (3) was proved by Ma and Minda in [MM]. Sugawa in his proof used also some property of the class $\mathcal{S}^{*}(\alpha)$ discovered by Fait, Krzyż and Zygmunt [FKZ]. In our case proving the equivalence of (4) and (6) we completed the theorem in an elementary way.

\section{References}

[BK] D. A. Brannan and W. E. Kirwan, On some classes of bounded univalent functions, J. London Math. Soc. 1 (1969), 431-443.

[BC] Cz. Bucka and K. Ciozda, On a new subclass of the class S, Ann. Polon. Math. 28 (1973), 153-161.

[FKZ] M. Fait, J. G. Krzyż and J. Zygmunt, Explicit quasiconformal extensions for some classes of univalent functions, Comment. Math. Helv. 51 (1976), 279-285.

[G] A. W. Goodman, Univalent Functions, Mariner, Tampa, FL, 1983.

[L] A. Lecko, On the class of functions strongly starlike of order $\alpha$ with respect to a point, Ann. Polon. Math. 68 (1998), 107-117.

[LL] A. Lecko and M. Lecko, On some subclasses of functions starlike with respect to the fixed point, Mathematica (Cluj) 39 (62) (1997), 245-256.

[LS] A. Lecko and J. Stankiewicz, An internal geometric characterization of some subclasses of starlike functions, in: XVIth Rolf Nevanlinna Colloquium (Joensuu, 1995), de Gruyter, Berlin, 1997, 231-238. 
[MM] W. Ma and D. Minda, An internal geometric characterization of strongly starlike functions, Ann. Univ. Mariae Curie-Skłodowska Sect. A 45 (1991), 89-97.

[P1] Ch. Pommerenke, Univalent Functions, Vandenhoeck and Ruprecht, Göttingen, 1975 .

[P2] - Boundary Behaviour of Conformal Maps, Springer, Berlin, 1992.

[S1] J. Stankiewicz, Quelques problèmes extrémaux dans les classes des fonctions $\alpha$ angulairement étoilées, Ann. Univ. Mariae Curie-Skłodowska Sect. A 20 (1966), $59-75$.

[S2] - On a family of starlike functions, ibid. 22-24 (1968-70), 175-181.

[SW] J. Stankiewicz and A. Wiśniowska, On geometric interpretations for some subclasses of univalent functions, Folia Sci. Univ. Tech. Res. 103 (1992), 5-9.

[Su] T. Sugawa, A self-duality of strong starlikeness, preprint.

[W] J. K. Wald, On starlike functions, Ph.D. thesis, Univ. of Delaware, 1978.

Department of Mathematics

Rzeszów University of Technology

W. Pola 2

35-959 Rzeszów, Poland

E-mail: alecko@prz.rzeszow.pl

Reçu par la Rédaction le 17.1.2005

Révisé le 11.4.2005 